\title{
Assessment of the local stress state through macroscopic variables
}

\author{
By ROBERT P. LIPTON \\ Department of Mathematics, Louisiana State University, \\ Baton Rouge, LA 70803, USA
}

Published online 27 March 2003

\begin{abstract}
Macroscopic quantities beyond effective elastic tensors are presented that can be used to assess the local state of stress within a composite in the linear elastic regime. These are presented in a general homogenization context. It is shown that the gradient of the effective elastic property can be used to develop a lower bound on the maximum pointwise equivalent stress in the fine-scale limit. Upper bounds are more sensitive and are correlated with the distribution of states of the equivalent stress in the finescale limit. The upper bounds are given in terms of the macrostress modulation function. This function gauges the magnitude of the actual stress. For $1 \leqslant p<$ $\infty$, upper bounds are found on the limit superior of the sequence of $L^{p}$ norms of stresses associated with discrete microstructure in the fine-scale limit. Conditions are given for which upper bounds can be found on the limit superior of the sequence of $L^{\infty}$ norms of stresses associated with the discrete microstructure in the fine-scale limit. For microstructure with oscillation on a sufficiently small scale we are able to give pointwise bounds on the actual stress in terms of the macrostress modulation function.
\end{abstract}

Keywords: composite materials; elastic stress analysis; microstructure

\section{Introduction}

Several technologically important applications have benefited from the use of graded composite materials (see Glasser 1997; Koizumi 1997). Graded materials provide the designer with the freedom to tailor the material's microstructure in order to enhance structural performance. In addition to designing for structural response, it is of vital importance to control the stress inside structural components made from composite materials. Regions of large stresses are most likely to be the first to exhibit failure during service.

Failure criteria such as the yield surface in plasticity require the knowledge of the stresses within each phase of the composite. However, graded composite materials are often made up of a very large number of discrete phases interspersed on a small scale. The direct evaluation of stress fields resulting from body or boundary loads lies beyond current experimental methods. A central theoretical problem understanding the asymptotic behaviour of the stress for linearly elastic composites in the limit of infinitely fine mixing of the discrete phases. The goal of this analysis is to identify

One contribution of 12 to a Theme 'Micromechanics of fluid suspensions and solid composites'. 
suitable macroscopic quantities that can be used to assess the state of stress within a composite.

In this treatment no assumption on the microstructure is made and the homogenized formula for the mean-square stress fluctuation is obtained (see theorem 2.3). It is shown that the formula provides a lower bound on the limit inferior of the sequence of $L^{\infty}$ norms of stresses associated with the discrete microstructure in the fine-scale limit (see theorem 2.4). Mean-square stress fluctuations appear in many contexts including bounds for nonlinear composites (Ponte Castañeda \& Suquet 1998) and estimates for failure surfaces for statistically homogeneous random composites (Buryachenko 2001).

To obtain upper bounds on $L^{p}$ norms $1 \leqslant p \leqslant \infty$, new quantities for the assessment of the actual stress are introduced: see (1.5), (3.4)-(3.7) and (4.9). These quantities are referred to as macrostress modulation functions. They measure the amplification or diminution of the macrostress by the underlying microgeometry. For simple hypotheses on the asymptotic distribution of states for the stress, these quantities are used to obtain upper bounds on the limit superior of the sequence of $L^{\infty}$ norms of stresses associated with the discrete microstructure in the fine-scale limit (see corollaries 3.5 and 4.6). For $1 \leqslant p<\infty$, corollaries 3.6 and 4.7 provide upper bounds on the limit superior of the sequence of $L^{p}$ norms of stresses associated with the discrete microstructure in the fine-scale limit. For sufficiently small $\varepsilon$, pointwise bounds on the actual stress are given in terms of the macrostress modulation function (see (1.15) and corollaries 3.4 and 4.5). These results follow from a homogenization constraint that correlates the macrostress modulation functions with the homogenized distribution of states for the stress (see theorems 3.1 and 4.2). We apply the homogenization constraint to assess the size of sets over which the local stress in the composite exceeds a prescribed value (see corollaries 3.2, 3.3, 4.3 and 4.4). The principal tool used to derive the constraint is a local formula for the derivative of effective properties given in theorem 2.2. The methods developed here can be applied to obtain analogous results for local flux and gradient fields in the contexts of heat conduction, DC electric transport, and problems of diffusion.

Composites made from $N$ linearly elastic materials are considered. The domain containing the composite is denoted by $\Omega$ and the stress tensor inside the composite at the point $\boldsymbol{x}$ is denoted by $\sigma^{\varepsilon}(\boldsymbol{x})$. Failure criteria are often given in terms of a coordinate invariant measure of the stress. In this treatment we consider the equivalent stress given by

$$
\Pi\left(\sigma^{\varepsilon}\right)=\Pi \sigma^{\varepsilon}: \sigma^{\varepsilon},
$$

where $\boldsymbol{\Pi}$ is a positive definite fourth rank tensor (see Tsai \& Hahn 1980). Examples of (1.1) include the Von Mises equivalent stress given by

$$
\Pi\left(\sigma^{\varepsilon}\right)=\frac{1}{2}\left[\left(\sigma_{11}^{\varepsilon}-\sigma_{22}^{\varepsilon}\right)^{2}+\left(\sigma_{22}^{\varepsilon}-\sigma_{33}^{\varepsilon}\right)^{2}+\left(\sigma_{11}^{\varepsilon}-\sigma_{33}^{\varepsilon}\right)^{2}\right]+3\left(\left(\sigma_{12}^{\varepsilon}\right)^{2}+\left(\sigma_{13}^{\varepsilon}\right)^{2}+\left(\sigma_{23}^{\varepsilon}\right)^{2}\right),
$$

and the magnitude of the stress given by $\Pi\left(\sigma^{\varepsilon}\right)=\left|\sigma^{\varepsilon}\right|^{2}=\sum_{i j=1}^{3}\left(\sigma_{i j}^{\varepsilon}\right)^{2}$. The set in the $i$ th phase where $\Pi\left(\sigma^{\varepsilon}\right)>t$ is denoted by $S_{t, i}^{\varepsilon}$. The stress distribution function $\lambda_{i}^{\varepsilon}(t)$ is the volume (measure) of $S_{t, i}^{\varepsilon}$. One of the principal results of this paper is theorem 3.1. It provides a constraint relating macroscopic stress modulation function and $S_{t, i}^{\varepsilon}$ in the limit of vanishing $\varepsilon$.

To motivate theorem 3.1 and the macrostress modulation function we consider a composite with uniform periodic microstructure contained in the unit cube $Q$. The 
local elastic tensor for a $Q$-periodic configuration of $N$ anisotropic elastic materials is denoted by $\boldsymbol{C}(\boldsymbol{y})$. Each phase has an elastic tensor specified by $\boldsymbol{A}_{i}$ and $\boldsymbol{C}(\boldsymbol{y})=\boldsymbol{A}_{i}$ in the $i$ th phase. The characteristic function of the $i$ th phase is written as $\chi_{i}(\boldsymbol{y})$ and takes a value of unity in the $i$ th phase and zero otherwise. No constraints are placed on the arrangement of the phases inside $Q$. Here the description of the effective elastic properties and local stress and strain are well known (see Milton 2002). For this case the strain $\epsilon(\boldsymbol{y})$ in the composite can be decomposed into the average strain $\bar{\epsilon}$ and a periodic fluctuation $\epsilon(\boldsymbol{w}(\boldsymbol{y}))$ where the periodic displacement $\boldsymbol{w}$ is the solution of

$$
-\operatorname{div}(\boldsymbol{C}(\boldsymbol{y})(\epsilon(\boldsymbol{w}(\boldsymbol{y}))+\bar{\epsilon}))=0 .
$$

The stress in the composite is $\sigma(\boldsymbol{y})=\boldsymbol{C}(\boldsymbol{y})(\epsilon(\boldsymbol{w}(\boldsymbol{y}))+\bar{\epsilon})$. The average stress is related to the average strain $\bar{\epsilon}$ by $\bar{\sigma}=\boldsymbol{C}^{\mathrm{E}} \bar{\epsilon}$. Here $\boldsymbol{C}^{\mathrm{E}}$ is the effective elastic tensor.

One is interested in the maximum equivalent stress in the $i$ th phase generated by subjecting $Q$ to the average stress $\bar{\sigma}$. Or, more generally, it is of interest to know whether the specimen is experiencing an equivalent stress greater than a prescribed value on a subdomain of non-zero volume. Thus a natural choice for stress assessment is the $L^{\infty}(Q)$ norm. The $L^{\infty}(Q)$ norm of a function $g$ denoted by $\|g\|_{L^{\infty}(Q)}$ is defined in the following way. Consider the set of points $S_{t}$ in $Q$ where $|g(\boldsymbol{y})|>t$. The $L^{\infty}(Q)$ norm of $g$ is defined to be the greatest lower bound on the parameter $t$ for which $S_{t}$ has zero volume (measure). The volume (measure) of the set $S_{t}$ is denoted by $\left|S_{t}\right|$. It is evident that if $t \geqslant\|g\|_{L^{\infty}(Q)}$ then $\left|S_{t}\right|=0$ and if $\|g\|_{L^{\infty}(Q)}>t$ then $\left|S_{t}\right|>0$. The $L^{\infty}$ norm is consistent with failure criteria that require the equivalent stress to exceed a critical value over a finite volume of the composite sample before it will fail.

The tensor $\boldsymbol{F}^{i}(\boldsymbol{y})$ is defined for every $3 \times 3$ tensor $\bar{\epsilon}$ by

$$
\boldsymbol{F}^{i}(\boldsymbol{y}) \bar{\epsilon}: \bar{\epsilon}=\chi_{i}(\boldsymbol{y}) \Pi(\sigma(\boldsymbol{y}))=\chi_{i}(\boldsymbol{y}) \boldsymbol{\Pi}\left(\boldsymbol{A}^{i}(\epsilon(\boldsymbol{w}(\boldsymbol{y}))+\bar{\epsilon})\right):\left(\boldsymbol{A}^{i}(\epsilon(\boldsymbol{w}(\boldsymbol{y}))+\bar{\epsilon})\right) .
$$

Set

$$
f^{i}\left(\boldsymbol{S}^{\mathrm{E}} \bar{\sigma}\right)=\left\|\boldsymbol{F}^{i}(\boldsymbol{y}) \boldsymbol{S}^{\mathrm{E}} \bar{\sigma}: \boldsymbol{S}^{\mathrm{E}} \bar{\sigma}\right\|_{L(Q)^{\infty}}=\left\|\chi_{i}(\boldsymbol{y}) \Pi(\sigma(\boldsymbol{y}))\right\|_{L(Q)^{\infty}},
$$

where $\boldsymbol{S}^{\mathrm{E}}$ is the effective compliance $\left(\boldsymbol{C}^{\mathrm{E}}\right)^{-1}$. The quantity $f^{i}\left(\boldsymbol{S}^{\mathrm{E}} \bar{\sigma}\right)$ is referred to as the macrostress modulation function and measures the amplification or diminution of $\bar{\sigma}$ by the microstructure. Next we consider $\varepsilon_{k}=1 / k$, periodic composites specified by the re-scaled elastic tensors $\left\{\boldsymbol{C}\left(\boldsymbol{x} / \varepsilon_{k}\right)\right\}_{k=1}^{\infty}$. The $Q$-periodic solution $\boldsymbol{w}^{\varepsilon_{k}}$ of

$$
-\operatorname{div}\left(\boldsymbol{C}\left(\boldsymbol{x} / \varepsilon_{k}\right)\left(\epsilon\left(\boldsymbol{w}^{\varepsilon_{k}}(\boldsymbol{x})\right)+\bar{\epsilon}\right)\right)=0
$$

is given by $\boldsymbol{w}^{\varepsilon_{k}}(\boldsymbol{x})=\varepsilon_{k} \boldsymbol{w}\left(\boldsymbol{x} / \varepsilon_{k}\right)$ and $\sigma^{\varepsilon_{k}}(\boldsymbol{x})=\sigma\left(\boldsymbol{x} / \varepsilon_{k}\right)$. For every $\varepsilon_{k}$-periodic composite it follows from the change of variables $\boldsymbol{y}=\boldsymbol{x} / \varepsilon_{k}$ that the modulation of the average stress by the microstructure is always given by $f^{i}\left(\boldsymbol{S}^{\mathrm{E}} \bar{\sigma}\right)$, i.e.

$$
f^{i}\left(\boldsymbol{S}^{\mathrm{E}} \bar{\sigma}\right)=\left\|\chi_{i}\left(\boldsymbol{x} / \varepsilon_{k}\right) \Pi\left(\sigma^{\varepsilon_{k}}(\boldsymbol{x})\right)\right\|_{L(Q)^{\infty}}
$$

and the stress distribution function is independent of $\varepsilon_{k}$, i.e.

$$
\lambda_{i}^{\varepsilon_{k}}(t)=\lambda_{i}^{1}(t)=\theta_{t, i}
$$

It is evident that for this special case the modulation of the average stress by the microstructure reduces to an analysis of the unit periodic composite. For this case, the homogenization constraint expressed in theorem 3.1 follows immediately from the definition of $f^{i}\left(\boldsymbol{S}^{\mathrm{E}} \bar{\sigma}\right)$. Indeed, from (1.5) it is clear that $t \geqslant f^{i}\left(\boldsymbol{S}^{\mathrm{E}} \bar{\sigma}\right)$ implies 
that $\theta_{t, i}=0$, and equivalently if $\theta_{t, i}>0$ it follows that $f^{i}\left(\boldsymbol{S}^{\mathrm{E}} \bar{\sigma}\right)>t$. This delivers the homogenization constraint given by

$$
\theta_{t, i}\left(f^{i}\left(\boldsymbol{S}^{\mathrm{E}} \bar{\sigma}\right)-t\right) \geqslant 0 .
$$

This is the specialization of theorem 3.1 to the case at hand. In the general context the macroscopic stress is not uniform and the microgeometry is not periodic and can change across the specimen. However, theorem 3.1 shows that a constraint of the form (1.9) holds and is given in terms of quantities $\tilde{f}^{i}$ and $\theta_{t, i}(\boldsymbol{x})$ that generalize $f^{i}\left(\boldsymbol{S}^{\mathrm{E}} \bar{\sigma}\right)$ and $\theta_{t, i}$.

To fix ideas and to give further motivation for theorem 3.1 we provide a generalization of (1.9) that holds for a composite with uniform periodic microstructure that is subjected to a non-uniform load. Here the uniformly periodic composite specimen $\Omega$ is subjected to a body load $\boldsymbol{f}$. The displacement $\boldsymbol{u}^{\varepsilon_{k}}$ vanishes on the specimen boundary. The $\varepsilon_{k}$-periodic microstructure is given by the re-scaling $\boldsymbol{C}^{\varepsilon_{k}}(\boldsymbol{x})=\boldsymbol{C}\left(\boldsymbol{x} / \varepsilon_{k}\right)$. The stress and displacement in the specimen are denoted by $\sigma^{\varepsilon_{k}}$ and $\boldsymbol{u}^{\varepsilon_{k}}$, respectively. The equation of elastic equilibrium is given by

$$
-\operatorname{div} \sigma^{\varepsilon_{k}}=\boldsymbol{f}
$$

The elastic strain $\epsilon\left(\boldsymbol{u}^{\varepsilon_{k}}\right)$ is related to the stress by $\sigma^{\varepsilon_{k}}=\boldsymbol{C}^{\varepsilon_{k}} \epsilon\left(\boldsymbol{u}^{\varepsilon_{k}}\right)$.

The fundamental feature that distinguishes this example from the previous one is that the sets $S_{t, i}^{\varepsilon_{k}}$ are no longer periodic and self similar. The self similarity is broken by the fact that the non-uniform load $\boldsymbol{f}$ and the specimen shape can be incommensurate with the periodicity of the microgeometry.

For the case at hand the constraint analogous to (1.9) is written in terms of the macroscopic or homogenized stress. From the theory of periodic homogenization (Bensoussan et al. 1978) one has that $\sigma^{\varepsilon_{k}}$ and $\epsilon\left(\boldsymbol{u}^{\varepsilon_{k}}\right)$ converge to the macroscopic stress $\sigma^{\mathrm{M}}$ and strain $\epsilon\left(\boldsymbol{u}^{\mathrm{M}}\right)$ as $\varepsilon_{k}$ goes to zero. The macroscopic displacement $\boldsymbol{u}^{\mathrm{M}}$ vanishes on the boundary and the macroscopic stress satisfies the equilibrium equation $-\operatorname{div} \sigma^{\mathrm{M}}=\boldsymbol{f}$. The stress and strain are related through the homogenized constitutive law

$$
\sigma^{\mathrm{M}}(\boldsymbol{x})=\boldsymbol{C}^{\mathrm{E}} \epsilon\left(\boldsymbol{u}^{\mathrm{M}}(\boldsymbol{x})\right) .
$$

Now substitute $\sigma^{\mathrm{M}}(\boldsymbol{x})$ for $\bar{\sigma}$ in (1.5) to write $f^{i}\left(\boldsymbol{S}^{\mathrm{E}} \sigma^{\mathrm{M}}(\boldsymbol{x})\right)$. In order to complete the description of the homogenization constraint, a suitable generalization of $\theta_{t, i}$ given by (1.8) is needed. The indicator function for the set $S_{t, i}^{\varepsilon_{k}}$ is written $\chi_{t, i}^{\varepsilon_{k}}$, taking a value of unity in $S_{t, i}^{\varepsilon_{k}}$ and zero outside. The distribution function can then be written as $\lambda_{i}^{\varepsilon_{k}}(t)=\int_{\Omega} \chi_{t, i}^{\varepsilon_{k}} \mathrm{~d} \boldsymbol{x}$. From the theory of weak convergence (see Evans 1990) there exists a (Lebesgue measurable) density $\theta_{t, i}(\boldsymbol{x})$ taking values in the interval $[0,1]$ such that $\lim _{k \rightarrow \infty} \lambda_{i}^{\varepsilon_{k}}(t)=\int_{\Omega} \theta_{t, i}(\boldsymbol{x}) \mathrm{d} x$. The density $\theta_{t, i}$ is the distribution of states of the equivalent stress $\Pi\left(\sigma^{\varepsilon_{k}}\right)$ in the limit of vanishing $\varepsilon_{k}$. Here $\partial_{t} \theta_{t, i}(\boldsymbol{x})$ is the asymptotic density of states of the equivalent stress in the 'homogenized' composite. An application of theorem 4.2 delivers the homogenization constraints

$$
\theta_{t, i}(\boldsymbol{x})\left(f^{i}\left(\boldsymbol{S}^{\mathrm{E}} \sigma^{\mathrm{M}}(\boldsymbol{x})\right)-t\right) \geqslant 0, \quad i=1, \ldots, N,
$$

which hold almost everywhere on $\Omega$. It is clear that (1.12) is the extension of (1.9) to situations where the macrostress is no longer uniform.

We outline the applications of the homogenization constraint (1.12), noting that identical results hold for the more general contexts of graded periodic microstructures 
and microstructures specified by G-converging elastic tensors. The results for the general cases are given in $\S \S 3$ and 4 . It is clear from $(1.12)$ that $\theta_{t, i}(\boldsymbol{x})=0$ on sets where

$$
t>f^{i}\left(\boldsymbol{S}^{\mathrm{E}} \sigma^{\mathrm{M}}(\boldsymbol{x})\right)
$$

We choose a subset $\omega$ of the specimen. Thus, if (1.13) holds almost everywhere on $\omega$, then the measure of the sets in the $i$ th phase where $\Pi\left(\sigma^{\varepsilon_{k}}\right)>t$ must vanish in the fine-scale limit. This is a special case of corollary 4.3 which applies to graded periodic composites. If

$$
t>f^{i}\left(\boldsymbol{S}^{\mathrm{E}} \sigma^{\mathrm{M}}(\boldsymbol{x})\right)
$$

for $i=1, \ldots, N$ on $\omega$, then one can pass to a subsequence if necessary to find for almost every point in $\omega$ and for every scale $\varepsilon_{k}$ below a critical scale $\varepsilon_{0}$ that

$$
\Pi\left(\sigma^{\varepsilon_{k}}(\boldsymbol{x})\right) \leqslant t
$$

Here the critical scale $\varepsilon_{0}$ can depend on the point $\boldsymbol{x}$ in $\omega$ (see corollary 4.5). This shows that a pointwise bound on the macrostress modulation function delivers a pointwise bound on the equivalent stress in the composite when the microstructure is sufficiently fine. Next we set

$$
\tilde{t}=\sup _{\boldsymbol{x} \in \omega}\left(\max _{i}\left\{f^{i}\left(\boldsymbol{S}^{\mathrm{E}}(\underline{\boldsymbol{A}}, \boldsymbol{x}) \sigma^{\mathrm{M}}(\boldsymbol{x})\right)\right\}\right) .
$$

From (1.15) it then follows that

$$
\Pi\left(\sigma^{\varepsilon_{j}}(\boldsymbol{x})\right) \leqslant \tilde{t}
$$

for every $\varepsilon_{j}<\varepsilon_{0}$ and for almost all points in $\omega$. As before, the critical scale $\varepsilon_{0}$ can depend upon $\boldsymbol{x}$. This result also holds in the context of graded periodic microstructures (see corollary 4.5) and also in the general homogenization context (see corollary 3.4).

Conversely, it follows from (1.12) that

$$
f^{i}\left(\boldsymbol{S}^{\mathrm{E}} \sigma^{\mathrm{M}}(\boldsymbol{x})\right) \geqslant t
$$

on sets where $\theta_{t, i}(\boldsymbol{x})>0$, and it is evident that $f^{i}\left(\boldsymbol{S}^{\mathrm{E}} \sigma^{\mathrm{M}}(\boldsymbol{x})\right)=\infty$ on sets $\omega$ for which $\theta_{t, i}>0$ for every $t>0$.

We write the indicator function $\chi_{i}^{\varepsilon_{k}}$ by taking a value of unity in the $i$ th phase and zero outside. The $L^{\infty}$ norm of a function $g$ over the sample $\Omega$ is denoted by $\|g\|_{\infty}$. We consider $\left\|\chi_{i}^{\varepsilon_{k}} \Pi\left(\sigma^{\varepsilon_{k}}\right)\right\|_{\infty}$. Since $\chi_{i}^{\varepsilon_{k}}$ takes a value of unity in the $i$ th phase and zero outside, $\left\|\chi_{i}^{\varepsilon_{k}} \Pi\left(\sigma^{\varepsilon_{k}}\right)\right\|_{\infty}$ delivers the $L^{\infty}$ norm of $\Pi\left(\sigma^{\varepsilon_{k}}\right)$ in the $i$ th phase. Suppose it is known that $\ell=\limsup _{k \rightarrow \infty}\left\|\chi_{i}^{\varepsilon_{k}} \Pi\left(\sigma^{\varepsilon_{k}}\right)\right\|_{\infty}<\infty$. Suppose further that for an interval $0<\delta<b$ the measure (volume) of the sets where $\theta_{\ell-\delta, i}>0$ does not vanish. We then have the upper bound given by

$$
\limsup _{k \rightarrow \infty}\left\|\chi_{i}^{\varepsilon_{k}} \Pi\left(\sigma^{\varepsilon_{k}}\right)\right\|_{\infty} \leqslant\left\|f^{i}\left(\boldsymbol{S}^{\mathrm{E}} \sigma^{\mathrm{M}}(\boldsymbol{x})\right)\right\|_{\infty} .
$$

This follows in a straightforward manner from (1.17); see corollaries 3.5 and 4.6.

Phil. Trans. R. Soc. Lond. A (2003) 
For $1 \leqslant p<\infty$ consider the $L^{p}$ norm $\|g\|_{p}=\left(\int_{\Omega}|g(\boldsymbol{x})|^{p} \mathrm{~d} \boldsymbol{x}\right)^{1 / p}$. Suppose that $\lim \sup _{k \rightarrow \infty}\left\|\chi_{i}^{\varepsilon_{k}} \Pi\left(\sigma^{\varepsilon_{k}}\right)\right\|_{\infty}<\infty$. It follows from the constraint (1.12) and an analysis identical to the proof of corollary 3.6 that

$$
\limsup _{k \rightarrow \infty}\left\|\chi_{i}^{\varepsilon_{k}} \Pi\left(\sigma^{\varepsilon_{k}}\right)\right\|_{p} \leqslant\left\|f^{i}\left(\boldsymbol{S}^{\mathrm{E}} \sigma^{\mathrm{M}}(\boldsymbol{x})\right)\right\|_{\infty}|\Omega|^{1 / p}
$$

for any $p, 1 \leqslant p<\infty$. A similar result is presented for the general case in corollary 3.6; see also corollary 4.7. It turns out that the homogenization constraint obtained in the general context of G-convergence is nearly identical to (1.12), except that it is given in terms of a sequence of periodic problems posed on the unit cube $Q$ (see theorem 3.1 and corollaries 3.2-3.6).

Recent work by Pagano \& Yuan (2000) analyses the effect of the discrete microgeometry at the free edge of a loaded two-ply laminate. Here one ply is composed of a unidirectional fibre composite and the other is composed of homogeneous material. When the composite ply is modelled as a homogenized material with an effective anisotropic stiffness, there is a skin effect at the free edge of the two-ply laminate. Here a dramatic amplification of the macrostress occurs inside a thin skin near the ply boundary (see Pagano \& Rybicky 1974). Such skin effects are found to appear in other problems of anisotropic solid mechanics (see Biot 1966). Pagano \& Yuan (2000) replace the homogenized composite near the boundary with a representative volume element (RVE) containing the discrete fibre structure. The RVE is subjected to the macrostress near the free edge. Their numerical calculations show that the discrete fibre phase modulates the macrostress. The modulated macrostress field exhibits much smaller growth at the free edge. It is seen that the modulated macrostress approximates the observed behaviour of the actual stress at the free edge in the fibre reinforced composite. The analysis given here shows that when the microstructure is sufficiently fine relative to the ply dimensions the maximum modulated macrostress delivers a rigorous upper bound on the actual stress near the free edge (see equations (1.16), (3.13) and (4.15)).

It should be pointed out that one of the principal features of the theory given here is that the choice of RVE (given by the unit cube $Q$ ) arises naturally from the theory and is not imposed as a hypothesis (see $\S \S 3$ and 4). Also the theory automatically fixes the type of macroscopic loading for the micromechanical boundary-value problem posed on the RVE. In the general context of G-convergence, a sequence of micromechanical boundary-value problems associated with a sequence of microgeometries is solved (see $\S 3 a$ ).

Lastly, we provide the sequence of ideas behind the homogenization constraint (1.12). The same set-up is used to establish the homogenization constraint in the more general settings in $\S \S 3$ and 4 . One starts with the inequality

$$
\chi_{t, i}^{\varepsilon_{k}} \Pi\left(\sigma^{\varepsilon_{k}}\right)-t \chi_{t, i}^{\varepsilon_{k}}>0 \text {. }
$$

This follows from the definition of the set $S_{t, i}^{\varepsilon_{k}}$. The method developed here treats the set $S_{t, i}^{\varepsilon_{k}}$ as a new material phase with material property $\boldsymbol{P}_{N+1}$. The piecewise constant elasticity tensor for this new composite is written as $\boldsymbol{C}^{\varepsilon_{k}}\left(\boldsymbol{A}_{1}, \ldots, \boldsymbol{A}_{N}, \boldsymbol{P}_{N+1}, \boldsymbol{x}\right)$. The elasticity tensor for the actual composite is recovered by setting $\boldsymbol{P}_{N+1}=\boldsymbol{A}_{i}$ in $S_{t, i}^{\varepsilon_{k}}$. Because the sets $S_{t, i}^{\varepsilon_{k}}$ are not $\varepsilon_{k}$-periodic, one cannot use periodic homogenization, but instead must work in the general homogenization context provided by the G-convergence (see Dal Maso 1993). The effective elasticity or G-limit for the 
sequence $\left\{\boldsymbol{C}^{\varepsilon_{k}}\left(\boldsymbol{A}_{1}, \ldots, \boldsymbol{A}_{N}, \boldsymbol{P}_{N+1}, \boldsymbol{x}\right)\right\}_{k=1}^{\infty}$ is denoted by $\widetilde{\boldsymbol{C}}^{\mathrm{E}}\left(\boldsymbol{A}_{1}, \ldots, \boldsymbol{P}_{N+1}, \boldsymbol{x}\right)$. The distributional limit of the sequence of products $\left\{\chi_{t, i}^{\varepsilon_{k}} \Pi\left(\sigma^{\varepsilon_{k}}\right)\right\}_{k=1}^{\infty}$ as $\varepsilon_{k}$ tends to zero is identified as

$$
\left(\boldsymbol{A}_{i} \boldsymbol{\Pi} \boldsymbol{A}_{i} \nabla^{N+1} \widetilde{\boldsymbol{C}}^{\mathrm{E}}\left(\boldsymbol{A}_{1}, \ldots, \boldsymbol{A}_{N}, \boldsymbol{A}_{i}, \boldsymbol{x}\right)\right) \boldsymbol{S}^{\mathrm{E}} \sigma^{\mathrm{M}}(\boldsymbol{x}): \boldsymbol{S}^{\mathrm{E}} \sigma^{\mathrm{M}}(\boldsymbol{x})
$$

(see theorem 2.3). Here $\nabla^{N+1} \widetilde{\boldsymbol{C}}^{\mathrm{E}}\left(\boldsymbol{A}_{1}, \ldots, \boldsymbol{A}_{N}, \boldsymbol{A}_{i}, \boldsymbol{x}\right)$ is the gradient of the G-limit $\widetilde{\boldsymbol{C}}^{\mathrm{E}}$ with respect to the variable $\boldsymbol{P}_{N+1}$ evaluated at $\boldsymbol{P}_{N+1}=\boldsymbol{A}_{i}$. It is shown in $\S 4$ that this limit is bounded above by $\theta_{t, i}(\boldsymbol{x}) f^{i}\left(\boldsymbol{S}^{\mathrm{E}} \sigma^{\mathrm{M}}(\boldsymbol{x})\right)$ for almost every $\boldsymbol{x}$ in the specimen. This bound follows from new explicit local formulae for the gradients of G-limits given in theorem 2.2 (see also Lipton 2003). The constraint (1.12) then follows by passing to the limit in the sense of distributions in (1.20) and applying the bound to the distributional limit of $\left\{\chi_{t, i}^{\varepsilon_{k}} \Pi\left(\sigma^{\varepsilon_{k}}\right)\right\}_{k=1}^{\infty}$ described above. In $\S 4$, inequalities analogous to (1.12) are established for the more general case of graded periodic microstructures in theorem 4.2. In $\S 3$ minimal hypotheses are placed on the microstructure and an inequality analogous to (1.12) is established in theorem 3.1. The minimal hypothesis is that the sequence of elastic tensors describing the microgeometry G converge as the scale of the microstructure tends to zero. It is pointed out that this hypothesis is weak in the sense that any sequence of configurations with elastic tensors taking values $\boldsymbol{A}_{i}$ in the $i$ th material is guaranteed to have a G-converging subsequence (see Spagnolo 1976; Murat \& Tartar 1997; Simon 1979).

In order to expedite the presentation, the following notation is used. The contractions of symmetric fourth-order tensors $\boldsymbol{A}$ with second-order tensors $\sigma^{\mathrm{M}}$ are written $[A]_{i j k l} \sigma_{k l}^{\mathrm{M}}=\boldsymbol{A} \sigma^{\mathrm{M}}$; contractions of two fourth-order tensors $\boldsymbol{A}$ and $\boldsymbol{M}$ are written $[A]_{i j k l}[M]_{k l o p}=\boldsymbol{A} \boldsymbol{M}$; contractions of two second-order tensors $\sigma, \epsilon$ are written $\sigma_{i j} \epsilon_{i j}=\sigma: \epsilon, \sigma: \sigma=|\sigma|^{2}$; and contractions of second-order tensors $\epsilon$ and vectors $\boldsymbol{x}$ are written $\epsilon_{i j} x_{j}=\epsilon \boldsymbol{x}$. The Lebesgue measure of a set $S$ is denoted by $|S|$.

\section{Homogenization of mean-square stress fluctuations and lower bounds on stress concentrations}

We consider graded composite materials made from $N$ linearly elastic phases. The elastic phases occupy the $N$ subsets $\Omega_{1}, \Omega_{2}, \ldots, \Omega_{N}$, where $\Omega_{1} \cup \Omega_{2} \cup \cdots \cup \Omega_{N}=$ $\Omega$. Here we make minimal assumptions on the shapes of the subsets and simply assume that they are Lebesgue measurable. The characteristic length-scale of the microgeometry relative to the size of the structure is denoted by $\varepsilon$. The local elasticity tensor $\boldsymbol{C}^{\varepsilon}(\underline{\boldsymbol{A}}, \boldsymbol{x})$ is piecewise constant and takes the value $\boldsymbol{C}^{\varepsilon}(\underline{\boldsymbol{A}}, \boldsymbol{x})=\boldsymbol{A}_{i}$ in the $i$ th phase. Denoting the indicator function of the $i$ th material by $\chi_{i}^{\varepsilon}$, the local elasticity tensor is written $\boldsymbol{C}^{\varepsilon}(\underline{\boldsymbol{A}}, \boldsymbol{x})=\sum_{i=1}^{N} \chi_{i}^{\varepsilon} \boldsymbol{A}_{i}$. Here $\chi_{i}^{\varepsilon}$ takes a value of unity for points inside the $i$ th phase and zero outside.

For a body load $\boldsymbol{f}$ the elastic displacement $\boldsymbol{u}^{\varepsilon}$ is the solution of

$$
-\operatorname{div}\left(\boldsymbol{C}^{\varepsilon}(\underline{\boldsymbol{A}}, \boldsymbol{x}) \epsilon\left(\boldsymbol{u}^{\varepsilon}(\boldsymbol{x})\right)\right)=\boldsymbol{f} .
$$

At each point in the composite the stress $\sigma^{\varepsilon}$ is given by the constitutive relation

$$
\sigma^{\varepsilon}(\boldsymbol{x})=\boldsymbol{C}^{\varepsilon}(\underline{\boldsymbol{A}}, \boldsymbol{x}) \epsilon\left(\boldsymbol{u}^{\varepsilon}(\boldsymbol{x})\right) .
$$

Here $\epsilon\left(\boldsymbol{u}^{\varepsilon}\right)$ is the strain tensor given by

$$
\epsilon\left(\boldsymbol{u}^{\varepsilon}\right)=\frac{1}{2}\left(u_{i, j}^{\varepsilon}+u_{j, i}^{\varepsilon}\right)
$$

Phil. Trans. R. Soc. Lond. A (2003) 
where $u_{i}^{\varepsilon}$ is the $i$ th component of displacement $\boldsymbol{u}^{\varepsilon}$ and $u_{i, j}^{\varepsilon}$ is its partial derivative in the $j$ th coordinate direction. The prescribed displacement on the boundary of the structure is zero. The solution $\boldsymbol{u}^{\varepsilon}$ is square integrable and has square-integrable derivatives. The space of such functions that vanish on the boundary is denoted by $H_{0}^{1}(\Omega)^{3}$. The dual space is written as $H^{-1}(\Omega)^{3}$ and the body force $\boldsymbol{f}$ is an element of this space (see Duvaut \& Lions 1976). It is assumed that the sequence of elastic tensors $\left\{\boldsymbol{C}^{\varepsilon}(\underline{\boldsymbol{A}}, \boldsymbol{x})\right\}_{\varepsilon>0}$ G-converge to the effective elastic tensor $\boldsymbol{C}^{\mathrm{E}}(\underline{\boldsymbol{A}}, \boldsymbol{x})$. The definition of G-convergence is given below (see Spagnolo 1976).

Definition 2.1 (G-convergence). The sequence $\left\{\boldsymbol{C}^{\varepsilon}(\underline{\boldsymbol{A}}, \boldsymbol{x})\right\}_{\varepsilon>0}$ is said to Gconverge to $\boldsymbol{C}^{\mathrm{E}}$ if, for every load $\boldsymbol{f}$ (in $H^{-1}(\Omega)^{3}$ ), the sequence of elastic displacements $\left\{\boldsymbol{u}^{\varepsilon}\right\}_{\varepsilon>0}$ converges weakly (in $H_{0}^{1}(\Omega)^{3}$ ) to $\boldsymbol{u}^{\mathrm{M}}$ where

$$
-\operatorname{div}\left(\boldsymbol{C}^{\mathrm{E}}(\underline{\boldsymbol{A}}, \boldsymbol{x}) \epsilon\left(\boldsymbol{u}^{\mathrm{M}}(\boldsymbol{x})\right)\right)=\boldsymbol{f} .
$$

The effective tensor or G-limit $\boldsymbol{C}^{\mathrm{E}}(\underline{\boldsymbol{A}}, \boldsymbol{x})$ depends upon $\boldsymbol{x}$ and is a measurable function (see Spagnolo 1976). Here the sequence of stresses $\left\{\sigma^{\varepsilon}\right\}_{\varepsilon>0}$ converges weakly (in $\left.L^{2}(\Omega)^{3 \times 3}\right)$ to the macroscopic stress $\sigma^{\mathrm{M}}$, and the macroscopic constitutive relation is given by

$$
\sigma^{\mathrm{M}}(\boldsymbol{x})=\boldsymbol{C}^{\mathrm{E}}(\underline{\boldsymbol{A}}, \boldsymbol{x}) \epsilon\left(\boldsymbol{u}^{\mathrm{M}}(\boldsymbol{x})\right) .
$$

The hypotheses necessary for $\mathrm{G}$-convergence are minimal. Indeed for any sequence $\left\{\boldsymbol{C}^{\varepsilon}(\boldsymbol{A}, \boldsymbol{x})\right\}_{\varepsilon>0}$ described above one is guaranteed the existence of a G-convergent subsequence (see Murat \& Tartar 1997; Simon 1979; Spagnolo 1976).

We start by computing the limit as $\varepsilon$ tends to zero of $\chi_{i}^{\varepsilon} \Pi\left(\sigma^{\varepsilon}\right)$ in the sense of distributions. We show that this limit is given in terms of derivatives of the effective tensor $\boldsymbol{C}^{\mathrm{E}}(\underline{\boldsymbol{A}}, \boldsymbol{x})$. In order to define derivatives of G-limits we introduce the neighbourhood $\underline{\mathcal{N}}(\underline{\boldsymbol{A}})$ of the array $\underline{\boldsymbol{A}}$. Arrays in this neighbourhood are denoted by $\underline{\boldsymbol{P}}=\left(\boldsymbol{P}_{1}, \boldsymbol{P}_{2}, \ldots, \boldsymbol{P}_{N}\right)$. The neighbourhood is chosen such that all tensors $\boldsymbol{P}_{i}$ in the array satisfy the constraint $0<\lambda<\boldsymbol{P}_{i}<\Lambda$. Here $\lambda$ and $\Lambda$ are bounds on the smallest and largest eigenvalues of the elasticity tensors.

The elasticity tensor associated with a particular choice of component elastic tensors is written

$$
\boldsymbol{C}^{\varepsilon}(\underline{\boldsymbol{P}}, \boldsymbol{x})=\sum_{i=1}^{N} \chi_{i}^{\varepsilon} \boldsymbol{P}_{i} \quad \text { and } \quad \boldsymbol{C}^{\varepsilon}(\underline{\boldsymbol{A}}, \boldsymbol{x})=\sum_{i=1}^{N} \chi_{i}^{\varepsilon} \boldsymbol{A}_{i} .
$$

Passing to a subsequence if necessary one is guaranteed that the G-limit $\boldsymbol{C}^{\mathrm{E}}(\underline{\boldsymbol{P}}, \boldsymbol{x})$ of $\left\{\boldsymbol{C}^{\varepsilon}(\underline{\boldsymbol{P}}, \boldsymbol{x})\right\}_{\varepsilon>0}$ exists for every $\underline{\boldsymbol{P}}$ in $\mathcal{N}(\underline{\boldsymbol{A}})$ (see Tartar 2000).

We introduce a sequence of cubes $Q(\boldsymbol{x}, r)$ of diameter $r$ containing a prescribed point $\boldsymbol{x}$ in $\Omega$. For $r$ sufficiently small, $Q(\boldsymbol{x}, r)$ is contained within $\Omega$. Given a constant strain $\bar{\epsilon}$ applied to the cube the local oscillatory response function is denoted by $\boldsymbol{w}_{\bar{\epsilon}}^{\varepsilon, r}$, where $\boldsymbol{w}_{\bar{\epsilon}}^{\varepsilon, r}$ is the $Q(\boldsymbol{x}, r)$-periodic solution of

$$
-\operatorname{div}\left(\boldsymbol{C}^{\varepsilon}(\underline{\boldsymbol{A}}, \boldsymbol{y})\left(\epsilon\left(\boldsymbol{w}_{\bar{\epsilon}}^{\varepsilon, r}(\boldsymbol{y})\right)+\bar{\epsilon}\right)\right)=0, \quad \text { for } \boldsymbol{y} \text { in } Q(\boldsymbol{x}, r) .
$$

The local response functions are used to define directional derivatives of the G-limit in the following theorem.

Theorem 2.2. The directional derivative of $\boldsymbol{C}^{\mathrm{E}}(\underline{\boldsymbol{P}}, \boldsymbol{x})$ at $\underline{\boldsymbol{P}}=\underline{\boldsymbol{A}}$ with respect to the $i$ th component elasticity in the direction specified by the symmetric fourth-order 
tensor $\boldsymbol{M}_{i}$ is given by

$$
\begin{aligned}
\frac{\partial \boldsymbol{C}^{\mathrm{E}}(\underline{\boldsymbol{A}}, \boldsymbol{x})}{\partial \boldsymbol{M}_{i}} \bar{\epsilon}: \bar{\epsilon} & =\boldsymbol{M}_{i} \nabla^{i} \boldsymbol{C}^{\mathrm{E}}(\underline{\boldsymbol{A}}, \boldsymbol{x}) \bar{\epsilon}: \bar{\epsilon} \\
= & \sum_{k=1}^{3} \sum_{l=1}^{3} \sum_{m=1}^{3} \sum_{n=1}^{3}\left[M_{i}\right]_{k l m n} \lim _{r \rightarrow 0} \lim _{\varepsilon \rightarrow 0}\left(\frac{1}{|Q(\boldsymbol{x}, r)|}\right) \\
& \times \int_{Q(\boldsymbol{x}, r)} \chi_{i}^{\varepsilon}\left(\epsilon\left(\boldsymbol{w}_{\bar{\epsilon}}^{\varepsilon, r}\right)_{k l}+\bar{\epsilon}_{k l}\right)\left(\epsilon\left(\boldsymbol{w}_{\bar{\epsilon}}^{\varepsilon, r}\right)_{m n}+\bar{\epsilon}_{m n}\right) \mathrm{d} \boldsymbol{y},
\end{aligned}
$$

where $\bar{\epsilon}$ is any constant strain. The derivative is Lebesgue measurable with respect to $\boldsymbol{x}$.

Here the $i$ th phase gradient $\nabla_{k l m n}^{i} \boldsymbol{C}^{\mathrm{E}}(\underline{\boldsymbol{A}}, \boldsymbol{x}) \bar{\epsilon}: \bar{\epsilon}$ is given by the local formulae

$$
\begin{aligned}
\nabla_{k l m n}^{i} \boldsymbol{C}^{\mathrm{E}}(\underline{\boldsymbol{A}}, \boldsymbol{x}) \bar{\epsilon}: \bar{\epsilon} \\
\quad=\lim _{r \rightarrow 0} \lim _{\varepsilon \rightarrow 0}\left(\frac{1}{|Q(\boldsymbol{x}, r)|}\right) \int_{Q(\boldsymbol{x}, r)} \chi_{i}^{\varepsilon}\left(\epsilon\left(\boldsymbol{w}_{\bar{\epsilon}}^{\varepsilon, r}\right)_{k l}+\bar{\epsilon}_{k l}\right)\left(\epsilon\left(\boldsymbol{w}_{\bar{\epsilon}}^{\varepsilon, r}\right)_{m n}+\bar{\epsilon}_{m n}\right) \mathrm{d} \boldsymbol{y}
\end{aligned}
$$

and

$$
\begin{aligned}
& \nabla_{k l k l}^{i} \boldsymbol{C}^{\mathrm{E}}(\underline{\boldsymbol{A}}, \boldsymbol{x}) \bar{\epsilon}: \bar{\epsilon} \\
& \quad=\lim _{r \rightarrow 0} \lim _{\varepsilon \rightarrow 0}\left(\frac{1}{|Q(\boldsymbol{x}, r)|}\right) \int_{Q(\boldsymbol{x}, r)} \chi_{i}^{\varepsilon}\left(\epsilon\left(\boldsymbol{w}_{\bar{\epsilon}}^{\varepsilon, r}\right)_{k l}+\bar{\epsilon}_{k l}\right)\left(\epsilon\left(\boldsymbol{w}_{\bar{\epsilon}}^{\varepsilon, r}\right)_{k l}+\bar{\epsilon}_{k l}\right) \mathrm{d} \boldsymbol{y}
\end{aligned}
$$

where repeated indices indicate summation. The formula for the G-limit is

$$
\boldsymbol{C}^{\mathrm{E}}(\underline{\boldsymbol{A}}, \boldsymbol{x}) \bar{\epsilon}: \bar{\epsilon}=\lim _{r \rightarrow 0} \lim _{\varepsilon \rightarrow 0}\left(\frac{1}{|Q(\boldsymbol{x}, r)|}\right) \int_{Q(\boldsymbol{x}, r)} \boldsymbol{C}^{\varepsilon}(\underline{\boldsymbol{A}}, \boldsymbol{y})\left(\epsilon\left(\boldsymbol{w}_{\bar{\epsilon}}^{\varepsilon, r}\right)+\bar{\epsilon}\right):\left(\epsilon\left(\boldsymbol{w}_{\bar{\epsilon}}^{\varepsilon, r}\right)+\bar{\epsilon}\right) \mathrm{d} \boldsymbol{y} .
$$

These formulae hold for any sequence of cubes $Q(\boldsymbol{x}, r)$ that contain $\boldsymbol{x}$ for every $r>0$. The local formula (2.10) for the G-limit was developed earlier (see Spagnolo 1976). The formulae (2.7) and (2.8) for the derivatives of the effective elastic tensor are semi-explicit in that they are given in terms of sequences of solutions to local problems. These formulae hold for general oscillations and are obtained without any hypotheses on the sequence of configurations.

The distributional limit of $\chi_{i}^{\varepsilon} \Pi\left(\sigma^{\varepsilon}\right)$ is given in the following theorem.

Theorem 2.3 (homogenization of stress fluctuations in the $i$ th phase). Given that $\left\{\boldsymbol{C}^{\varepsilon}(\underline{\boldsymbol{A}}, \boldsymbol{x})\right\}_{\varepsilon>0}$ G-converges to $\boldsymbol{C}^{\mathrm{E}}(\underline{\boldsymbol{A}}, \boldsymbol{x})$, as $\varepsilon$ tends to zero

$$
\chi_{i}^{\varepsilon} \Pi\left(\sigma^{\varepsilon}(\boldsymbol{x})\right) \rightarrow \boldsymbol{\Pi}^{H, i}(\boldsymbol{x}) \sigma^{\mathrm{M}}(\boldsymbol{x}): \sigma^{\mathrm{M}}(\boldsymbol{x})
$$

in the sense of distributions and

$$
\boldsymbol{\Pi}^{H, i}(\boldsymbol{x}) \sigma^{\mathrm{M}}(\boldsymbol{x}): \sigma^{\mathrm{M}}(\boldsymbol{x})=\left(\boldsymbol{S}^{\mathrm{E}}(\underline{\boldsymbol{A}}, \boldsymbol{x}) \boldsymbol{A}_{i} \boldsymbol{\Pi} \boldsymbol{A}_{i} \nabla^{i} \boldsymbol{C}^{\mathrm{E}}(\underline{\boldsymbol{A}}, \boldsymbol{x}) \boldsymbol{S}^{\mathrm{E}}(\underline{\boldsymbol{A}}, \boldsymbol{x})\right) \sigma^{\mathrm{M}}(\boldsymbol{x}): \sigma^{\mathrm{M}}(\boldsymbol{x}) .
$$

Here $\boldsymbol{S}^{\mathrm{E}}(\underline{\boldsymbol{A}}, \boldsymbol{x})=\left(\boldsymbol{C}^{\mathrm{E}}\right)^{-1}(\underline{\boldsymbol{A}}, \boldsymbol{x})$ is the effective compliance. 
Passing to a subsequence if necessary, one finds that $\left\{\chi_{i}^{\varepsilon}\right\}_{\varepsilon>0}$ converges weakly to a density $\theta_{i}(\boldsymbol{x})$, such that for any cube $Q(\boldsymbol{x}, r)$ in $\Omega$ one has

$$
\lim _{\varepsilon \rightarrow 0} \int_{Q(\boldsymbol{x}, r)}\left(\chi_{i}^{\varepsilon}(\boldsymbol{y})-\theta_{i}(\boldsymbol{y})\right) \mathrm{d} \boldsymbol{y}=0
$$

(see Evans 1990). This convergence is written as $\chi_{i}^{\varepsilon} \stackrel{*}{\rightarrow} \theta_{i}$ in $L^{\infty}(\Omega)$.

The next result gives a lower bound on the stress concentrations in the limit $\varepsilon=0$.

Theorem 2.4. Given that $\left\{\boldsymbol{C}^{\varepsilon}(\underline{\boldsymbol{A}}, \boldsymbol{x})\right\}_{\varepsilon>0}$ G-converges to $\boldsymbol{C}^{\mathrm{E}}(\underline{\boldsymbol{A}}, \boldsymbol{x}), \chi_{i}^{\varepsilon} \stackrel{*}{\rightarrow} \theta_{i}$,

$$
\boldsymbol{\Pi}^{H, i}(\boldsymbol{x}) \sigma^{\mathrm{M}}(\boldsymbol{x}): \sigma^{\mathrm{M}}(\boldsymbol{x}) \leqslant \theta_{i}(\boldsymbol{x}) \liminf _{\varepsilon \rightarrow 0}\left\|\chi_{i}^{\varepsilon} \Pi\left(\sigma^{\varepsilon}\right)\right\|_{\infty},
$$

almost everywhere. One also has the following theorem.

Theorem 2.5. Given that $\left\{\boldsymbol{C}^{\varepsilon}(\underline{\boldsymbol{A}}, \boldsymbol{x})\right\}_{\varepsilon>0}$ G-converges to $\boldsymbol{C}^{\mathrm{E}}(\underline{\boldsymbol{A}}, \boldsymbol{x})$,

$$
\left\|\sum_{i=1}^{N} \Pi^{H, i} \sigma^{\mathrm{M}}: \sigma^{\mathrm{M}}\right\|_{\infty} \leqslant \liminf _{\varepsilon \rightarrow 0}\left\|\Pi\left(\sigma^{\varepsilon}\right)\right\|_{\infty} .
$$

An illustrative example of the lower bound is given for a long shaft subjected to torsion loading. Here the cross-section of the shaft is composed of a periodic chequerboard pattern of two materials of shear stiffness $G_{1}$ and $G_{2}$, and we choose $\Pi(\sigma)=|\sigma|^{2}$. For torsion loading, the non-vanishing components of stress are $\sigma_{13}^{\varepsilon}$, $\sigma_{23}^{\varepsilon}$. One has

$$
\sum_{i=1}^{2} \Pi^{H, i}=\frac{G_{1}+G_{2}}{2 \sqrt{G_{1} G_{2}}}
$$

and

$$
\frac{G_{1}+G_{2}}{2 \sqrt{G_{1} G_{2}}}\left\|\left(\sigma_{13}^{\mathrm{M}}\right)^{2}+\left(\sigma_{23}^{\mathrm{M}}\right)^{2}\right\|_{\infty} \leqslant \liminf _{\varepsilon \rightarrow 0}\left\|\left(\sigma_{13}^{\varepsilon}\right)^{2}+\left(\sigma_{23}^{\varepsilon}\right)^{2}\right\|_{\infty} .
$$

It is clear from this example that the effect of the tensor $\sum_{i=1}^{2} \boldsymbol{\Pi}^{H, i}$ is significant, as it diverges when either shear modulus tends to zero.

The proofs of theorems $2.2-2.5$ are given in the following subsection.

\section{(a) Proofs of theorems 2.2-2.5}

First theorem 2.2 is established. Consider a sequence $\left\{\boldsymbol{C}^{\varepsilon}(\underline{\boldsymbol{P}}, \boldsymbol{x})\right\}_{\varepsilon>0}$ that Gconverges to $\boldsymbol{C}^{\mathrm{E}}(\underline{\boldsymbol{P}}, \boldsymbol{x})$ for every $\underline{\boldsymbol{P}}$ in $\mathcal{N}(\underline{\boldsymbol{A}})$. Given a constant strain $\bar{\epsilon}$ the local oscillatory response $\boldsymbol{w}_{\bar{\epsilon}}^{\varepsilon, r}$ is the $\left.\overline{Q(\boldsymbol{x}}, r\right)$ periodic solution of (2.6). The differential equation (2.6) is written in the weak form given by

$$
\int_{Q(\boldsymbol{x}, r)} \boldsymbol{C}^{\varepsilon}(\underline{\boldsymbol{A}}, \boldsymbol{y})\left(\epsilon\left(\boldsymbol{w}_{\bar{\epsilon}}^{\varepsilon, r}\right)+\bar{\epsilon}\right): \epsilon(\boldsymbol{v}) \mathrm{d} \boldsymbol{y}=0,
$$

for all $Q(\boldsymbol{x}, r)$ periodic $\boldsymbol{v}$ that are square integrable and have square-integrable partial derivatives. The volume of $Q(\boldsymbol{x}, r)$ is denoted by $|Q(\boldsymbol{x}, r)|$, and an application of Cauchy's inequality in (2.17) gives

$$
\int_{Q(\boldsymbol{x}, r)}\left|\epsilon\left(\boldsymbol{w}_{\bar{\epsilon}}^{\varepsilon, r}\right)\right|^{2} \mathrm{~d} \boldsymbol{y} \leqslant\left(\Lambda^{2} / \lambda^{2}\right)|Q(\boldsymbol{x}, r) \| \bar{\epsilon}|^{2}
$$


and

$$
\int_{Q(\boldsymbol{x}, r)}\left|\epsilon\left(\boldsymbol{w}_{\bar{\epsilon}}^{n}\right)+\bar{\epsilon}\right|^{2} \mathrm{~d} \boldsymbol{y} \leqslant\left(\Lambda^{2} / \lambda^{2}+1\right)|Q(\boldsymbol{x}, r)||\bar{\epsilon}|^{2} .
$$

Choose a number $\delta \beta$ and $\underline{\boldsymbol{M}}$ such that $\underline{\boldsymbol{A}}+\delta \beta \underline{\boldsymbol{M}}$ are in $\mathcal{N}(\underline{\boldsymbol{A}})$ and consider the sequence $\left\{\boldsymbol{C}^{\varepsilon}(\underline{\boldsymbol{A}}+\delta \beta \underline{\boldsymbol{M}}, x)\right\}_{\varepsilon>0}$. Here $\underline{\boldsymbol{M}}$ is an array of elastic tensors that is identically zero except for the $i$ th component elastic tensor $\boldsymbol{M}_{i}$. Here $\boldsymbol{M}_{i}$ is of norm one, where the tensor norm is given by

$$
\left|\boldsymbol{M}_{i}\right|=\sqrt{\sum_{k, l, m, n}\left[M_{i}\right]_{k l m n}^{2}} .
$$

The sequence of coefficients $\left\{\boldsymbol{C}^{\varepsilon}(\underline{\boldsymbol{A}}+\delta \beta \underline{\boldsymbol{M}}, \boldsymbol{x})\right\}_{\varepsilon>0}$ differ from $\left\{\boldsymbol{C}^{\varepsilon}(\underline{\boldsymbol{A}}, x)\right\}_{\varepsilon>0}$ by the increment $\delta \beta \boldsymbol{M}_{i} \chi_{i}^{\varepsilon}$. The G-limit for the sequence $\left\{\boldsymbol{C}^{\varepsilon}(\underline{\boldsymbol{A}}+\delta \beta \underline{\boldsymbol{M}}, \boldsymbol{x})\right\}_{\varepsilon>0}$ is written as $\boldsymbol{C}^{\mathrm{E}}(\underline{\boldsymbol{A}}+\delta \beta \underline{\boldsymbol{M}}, \boldsymbol{x})$. We set $\delta \boldsymbol{C}=\boldsymbol{C}^{\mathrm{E}}(\underline{\boldsymbol{A}}+\delta \beta \underline{\boldsymbol{M}}, \boldsymbol{x})-C^{\mathrm{E}}(\underline{\boldsymbol{A}}, \boldsymbol{x})$ and use $(2.10)$ to compute $\delta \boldsymbol{C}$ with respect to the increment $\delta \beta$. The oscillatory responses associated with the sequence $\left\{\boldsymbol{C}^{\varepsilon}(\underline{\boldsymbol{A}}+\delta \beta \underline{\boldsymbol{M}}, \boldsymbol{x})\right\}_{\varepsilon>0}$ are denoted by $\hat{\boldsymbol{w}}_{\bar{\epsilon}}^{\varepsilon, r}$, where $\hat{\boldsymbol{w}}_{\bar{\epsilon}}^{\varepsilon, r}$ are $Q(\boldsymbol{x}, r)$-periodic solutions of

$$
\int_{Q(\boldsymbol{x}, r)} \boldsymbol{C}^{\varepsilon}(\underline{\boldsymbol{A}}+\delta \beta \underline{\boldsymbol{M}}, \boldsymbol{y})\left(\epsilon\left(\hat{\boldsymbol{w}}_{\bar{\epsilon}}^{\varepsilon, r}\right)+\bar{\epsilon}\right): \epsilon(\boldsymbol{v}) \mathrm{d} \boldsymbol{y}=0
$$

for all $Q(\boldsymbol{x}, r)$-periodic $\boldsymbol{v}$ that are square integrable and have square-integrable derivatives.

It follows from (2.10) that

$\delta \boldsymbol{C} \bar{\epsilon}: \bar{\epsilon}$

$$
\begin{aligned}
= & \lim _{r \rightarrow 0} \lim _{\varepsilon \rightarrow 0}\left(\frac{1}{|Q(\boldsymbol{x}, r)|}\right) \int_{Q(\boldsymbol{x}, r)} \boldsymbol{C}^{\varepsilon}(\underline{\boldsymbol{A}}+\delta \beta \underline{\boldsymbol{M}}, \boldsymbol{y})\left(\epsilon\left(\hat{\boldsymbol{w}}_{\bar{\epsilon}}^{\varepsilon, r}\right)+\bar{\epsilon}\right):\left(\epsilon\left(\hat{\boldsymbol{w}}_{\bar{\epsilon}}^{\varepsilon, r}\right)+\bar{\epsilon}\right) \mathrm{d} \boldsymbol{y} \\
& \left.-\lim _{r \rightarrow 0} \lim _{\varepsilon \rightarrow 0}\left(\frac{1}{|Q(\boldsymbol{x}, r)|}\right) \int_{Q(\boldsymbol{x}, r)} \boldsymbol{C}^{\varepsilon}(\underline{\boldsymbol{A}}, \boldsymbol{y})\left(\epsilon\left(\boldsymbol{w}_{\bar{\epsilon}}^{\varepsilon, r}\right)+\bar{\epsilon}\right):\left(\boldsymbol{w}_{\bar{\epsilon}}^{\varepsilon, r}\right)+\bar{\epsilon}\right) \mathrm{d} \boldsymbol{y} .
\end{aligned}
$$

Writing

$$
\boldsymbol{C}^{\varepsilon}(\underline{\boldsymbol{A}}+\delta \beta \underline{\boldsymbol{M}}, \boldsymbol{y})=\boldsymbol{C}^{\varepsilon}(\underline{\boldsymbol{A}}, \boldsymbol{y})+\delta \beta \boldsymbol{M}_{i} \chi_{i}^{\varepsilon} \quad \text { and } \quad \hat{\boldsymbol{w}}_{\bar{\epsilon}}^{\varepsilon, r}=\boldsymbol{w}_{\bar{\epsilon}}^{\varepsilon, r}+\delta \boldsymbol{w}^{\varepsilon, r},
$$

where $\delta \boldsymbol{w}^{\varepsilon, r}=\hat{\boldsymbol{w}}_{\bar{\epsilon}}^{\varepsilon, r}-\boldsymbol{w}_{\bar{\epsilon}}^{\varepsilon, r}$, one has, for every $Q$-periodic $\boldsymbol{v}$, the equation

$$
\begin{aligned}
0=\int_{Q(\boldsymbol{x}, r)} \delta \beta \boldsymbol{M}_{i} \chi_{i}^{\varepsilon}\left(\epsilon\left(\boldsymbol{w}_{\bar{\epsilon}}^{\varepsilon, r}\right)+\bar{\epsilon}\right): \epsilon(\boldsymbol{v}) \mathrm{d} \boldsymbol{y} & \\
& +\int_{Q(\boldsymbol{x}, r)} \boldsymbol{C}^{\varepsilon}(\underline{\boldsymbol{A}}+\delta \beta \underline{\boldsymbol{M}}, \boldsymbol{y}) \epsilon\left(\delta \boldsymbol{w}^{\varepsilon, r}\right): \epsilon(\boldsymbol{v}) \mathrm{d} \boldsymbol{y} .
\end{aligned}
$$

Setting $\boldsymbol{v}=\delta \boldsymbol{w}^{\varepsilon, r}$ in (2.22) and substitution into (2.21) gives

$$
\begin{aligned}
\delta \boldsymbol{C} \bar{\epsilon}: \bar{\epsilon}=\delta & \beta \lim _{r \rightarrow 0} \lim _{\varepsilon \rightarrow 0}\left(\frac{1}{|Q(\boldsymbol{x}, r)|}\right) \int_{Q(\boldsymbol{x}, r)} \boldsymbol{M}_{i} \chi_{i}^{\varepsilon}\left(\epsilon\left(\boldsymbol{w}_{\bar{\epsilon}}^{\varepsilon, r}\right)+\bar{\epsilon}\right):\left(\epsilon\left(\boldsymbol{w}_{\bar{\epsilon}}^{\varepsilon, r}\right)+\bar{\epsilon}\right) \mathrm{d} \boldsymbol{y} \\
& +\delta \beta \lim _{r \rightarrow 0} \lim _{\varepsilon \rightarrow 0}\left(\frac{1}{|Q(\boldsymbol{x}, r)|}\right) \int_{Q(\boldsymbol{x}, r)} \boldsymbol{M}_{i} \chi_{i}^{\varepsilon}\left(\epsilon\left(\boldsymbol{w}_{\bar{\epsilon}}^{\varepsilon, r}\right)+\bar{\epsilon}\right): \epsilon\left(\delta \boldsymbol{w}^{\varepsilon, r}\right) \mathrm{d} \boldsymbol{y} .
\end{aligned}
$$


Setting $\boldsymbol{v}=\delta \boldsymbol{w}^{\varepsilon, r}$ in (2.22) and application of Cauchy's inequality gives

$$
\int_{Q(\boldsymbol{x}, r)}\left|\epsilon\left(\delta \boldsymbol{w}^{\varepsilon, r}\right)\right|^{2} \mathrm{~d} \boldsymbol{y} \leqslant \delta \beta^{2} \lambda^{-2}|Q(\boldsymbol{x}, r)||\bar{\epsilon}|^{2}\left(1+(\Lambda / \lambda)^{2}\right) .
$$

Estimates (2.19) and (2.24) show that

$$
\left|\left(\frac{1}{|Q(\boldsymbol{x}, r)|}\right) \int_{Q(\boldsymbol{x}, r)} \boldsymbol{M}_{i} \chi_{i}^{\varepsilon}\left(\epsilon\left(\boldsymbol{w}_{\bar{\epsilon}}^{\varepsilon, r}\right)+\bar{\epsilon}\right): \epsilon\left(\delta \boldsymbol{w}^{\varepsilon, r}\right) \mathrm{d} \boldsymbol{y}\right| \leqslant \delta \beta|\bar{\epsilon}|^{2}\left(1+(\Lambda / \lambda)^{2}\right) .
$$

From this, one deduces that

$\delta \boldsymbol{C} \bar{\epsilon}: \bar{\epsilon}=\delta \beta \lim _{r \rightarrow 0} \lim _{\varepsilon \rightarrow 0}\left(\frac{1}{|Q(\boldsymbol{x}, r)|}\right) \int_{Q(\boldsymbol{x}, r)} \boldsymbol{M}_{i} \chi_{i}^{\varepsilon}\left(\epsilon\left(\boldsymbol{w}_{\bar{\epsilon}}^{\varepsilon, r}\right)+\bar{\epsilon}\right):\left(\epsilon\left(\boldsymbol{w}_{\bar{\epsilon}}^{\varepsilon, r}\right)+\bar{\epsilon}\right) \mathrm{d} \boldsymbol{y}+o(\delta \beta)$

and (2.7) follows. The measurability of $\partial \boldsymbol{C}^{\mathrm{E}}(\underline{\boldsymbol{A}}, \boldsymbol{x}) / \partial \boldsymbol{M}_{i}$ is assured as it is a pointwise limit of measurable functions and theorem 2.2 is proven.

Theorem 2.3 follows from arguments analogous to those presented in Lipton (2002, 2003).

To establish theorem 2.4 note that for any positive test function $p$ in $C^{\infty}(\bar{\Omega})$ Hölder's inequality gives

$$
\int_{\Omega} p(\boldsymbol{x})\left(\chi_{i}^{\varepsilon} \Pi\left(\sigma^{\varepsilon}\right)-\chi_{i}^{\varepsilon}\left\|\chi_{i}^{\varepsilon} \Pi\left(\sigma^{\varepsilon}\right)\right\|_{\infty}\right) \mathrm{d} \boldsymbol{x} \leqslant 0 .
$$

Sending $\varepsilon$ to zero, together with theorem 2.3 , gives

$$
\int_{\Omega} p(\boldsymbol{x})\left(\boldsymbol{\Pi}^{H, i}(\boldsymbol{x}) \sigma^{\mathrm{M}}(\boldsymbol{x}): \sigma^{\mathrm{M}}(\boldsymbol{x})-\theta_{i}(\boldsymbol{x}) \liminf _{\varepsilon \rightarrow 0}\left\|\chi_{i}^{\varepsilon} \Pi\left(\sigma^{\varepsilon}\right)\right\|_{\infty}\right) \mathrm{d} \boldsymbol{x} \leqslant 0,
$$

for every choice of $p$ and theorem 2.4 follows. Theorem 2.5 also follows from Hölder's inequality.

\section{A homogenized constraint condition and local bounds on stress}

In this section homogenized quantities are introduced that provide information about the size of sets where the actual stress is large. We consider a sequence of elastic tensors $\left\{\boldsymbol{C}^{\varepsilon_{j}}(\underline{\boldsymbol{A}}, \boldsymbol{x})\right\}_{j=0}^{\infty}$, where the size of the microgeometry relative to the domain is given by $\varepsilon_{j}, j=1,2, \ldots$, and $\varepsilon_{j}$ tends to zero as $j$ goes to infinity. Here

$$
\boldsymbol{C}^{\varepsilon_{j}}(\underline{\boldsymbol{A}}, \boldsymbol{x})=\sum_{i=1}^{N} \chi_{i}^{\varepsilon_{j}} \boldsymbol{A}_{i}
$$

where $\chi_{i}^{\varepsilon_{j}}$ is the characteristic function of the $i$ th phase. We suppose that the sequence $\left\{\boldsymbol{C}^{\varepsilon_{j}}(\underline{\boldsymbol{A}}, \boldsymbol{x})\right\}_{j=0}^{\infty}$ G-converges to the effective elastic tensor $\boldsymbol{C}^{\mathrm{E}}(\underline{\boldsymbol{A}}, \boldsymbol{x})$. A body load $\boldsymbol{f}$ is applied to the domain $\Omega$, and the elastic displacements $\boldsymbol{u}^{\varepsilon_{j}}$ are solutions of

$$
-\operatorname{div}\left(\boldsymbol{C}^{\varepsilon_{j}}(\underline{\boldsymbol{A}}, \boldsymbol{x}) \epsilon\left(\boldsymbol{u}^{\varepsilon_{j}}(\boldsymbol{x})\right)\right)=\boldsymbol{f},
$$

with $\boldsymbol{u}^{\varepsilon_{j}}=0$ on the boundary of $\Omega$. The actual stress in the composite satisfies $\sigma^{\varepsilon_{j}}=\boldsymbol{C}^{\varepsilon_{j}}(\underline{\boldsymbol{A}}, \boldsymbol{x}) \epsilon\left(\boldsymbol{u}^{\varepsilon_{j}}(\boldsymbol{x})\right)$. 
The stresses $\left\{\sigma^{\varepsilon_{j}}\right\}_{j=1}^{\infty}$ converge weakly in $L^{2}(\Omega)^{3 \times 3}$ to the homogenized stress $\sigma^{\mathrm{M}}$ given by $\sigma^{\mathrm{M}}=\boldsymbol{C}^{\mathrm{E}}(\underline{\boldsymbol{A}}, x) \epsilon\left(\boldsymbol{u}^{\mathrm{M}}\right)$, and the displacements $\left\{\boldsymbol{u}^{\varepsilon_{j}}\right\}_{j=1}^{\infty}$ converge weakly to $\boldsymbol{u}^{\mathrm{M}}\left(\right.$ in $\left.H_{0}^{1}(\Omega)^{3}\right)$. Here $\boldsymbol{u}^{\mathrm{M}}$ is the solution of the homogenized problem given by

$$
-\operatorname{div}\left(\boldsymbol{C}^{\mathrm{E}}(\underline{\boldsymbol{A}}, \boldsymbol{x}) \epsilon\left(\boldsymbol{u}^{\mathrm{M}}\right)\right)=\boldsymbol{f}
$$

and $\boldsymbol{u}^{\mathrm{M}}=0$ on the boundary of $\Omega$. The effective compliance is given by $\left(\boldsymbol{C}^{\mathrm{E}}(\underline{\boldsymbol{A}}, x)\right)^{-1}$ and is denoted by $\boldsymbol{S}^{\mathrm{E}}(\underline{\boldsymbol{A}}, x)$.

We track the behaviour of the local stresses in each phase in the fine-scale limit. Consider the set $S_{t, i}^{\varepsilon_{j}}$ of points in the $i$ th phase where $\Pi\left(\sigma^{\varepsilon_{j}}\right)>t$. Choose a subset $\omega$ of the sample. Passing to a subsequence if necessary one has a density $\theta_{t, i}(\boldsymbol{x})$ taking values in the unit interval for which

$$
\lim _{j \rightarrow \infty}\left|S_{t, i}^{\varepsilon_{j}} \cap \omega\right|=\int_{\omega} \theta_{t, i} \mathrm{~d} \boldsymbol{x} .
$$

Here $\theta_{t, i}$ gives the asymptotic distribution of states for the equivalent stress $\Pi\left(\sigma^{\varepsilon_{j}}\right)$ in the limit of vanishing $\varepsilon_{j}$. It is evident that if $\theta_{t, i}=0$ on $\omega$ then the volume (measure) of the sets in $\omega$ where the equivalent stress exceeds $t$ in the $i$ th phase vanishes with $\varepsilon_{j}$. We provide a link between $\theta_{t, i}$ and the macroscopic stress $\sigma^{\mathrm{M}}$ appearing in the homogenized composite. This link is given by the macrostress modulation function. In this section the function is defined in the context of G-convergence. For the simpler case of periodic homogenization the macrostress modulation function is given by equation (1.5).

Consider a sequence of cubes $Q\left(\boldsymbol{x}, r_{j}\right)$ centred at a point $\boldsymbol{x}$ in $\Omega$ together with the values of $\boldsymbol{C}^{\varepsilon_{j}}(\underline{\boldsymbol{A}}, \boldsymbol{y})$ for points $\boldsymbol{y}$ in $Q\left(\boldsymbol{x}, r_{j}\right)$. It is assumed that $r_{j} \rightarrow 0$ as $j$ goes to infinity and $\varepsilon_{j} / r_{j} \rightarrow 0$. On re-scaling one considers $\boldsymbol{C}^{\varepsilon_{j}}\left(\underline{\boldsymbol{A}}, \boldsymbol{x}+r_{j} \boldsymbol{z}\right)$ for $\boldsymbol{z}$ in the unit cube $Q$ centred at the origin and

$$
\boldsymbol{C}^{\varepsilon_{j}}\left(\underline{\boldsymbol{A}}, \boldsymbol{x}+r_{j} \boldsymbol{z}\right)=\sum_{i=1}^{N} \chi_{i}^{\varepsilon_{j}}\left(\boldsymbol{x}+\boldsymbol{r}_{j} \boldsymbol{z}\right) \boldsymbol{A}_{i} .
$$

We introduce the tensor $\boldsymbol{F}_{j}^{i}(\boldsymbol{x}, \boldsymbol{z})$ defined on $\Omega \times Q$ such that for every constant symmetric $3 \times 3$ tensor $\bar{\epsilon}$ it is given by

$$
\begin{aligned}
\boldsymbol{F}_{j}^{i}(\boldsymbol{x}, \boldsymbol{z}) \bar{\epsilon}: \bar{\epsilon} & =\chi_{i}^{\varepsilon_{j}}\left(\boldsymbol{x}+r_{j} \boldsymbol{z}\right) \boldsymbol{\Pi} \boldsymbol{A}_{i}\left(\epsilon_{\boldsymbol{z}}\left(\boldsymbol{w}^{j}(\boldsymbol{x}, \boldsymbol{z})\right)+\bar{\epsilon}\right): \boldsymbol{A}_{i}\left(\epsilon_{\boldsymbol{z}}\left(\boldsymbol{w}^{j}(\boldsymbol{x}, \boldsymbol{z})\right)+\bar{\epsilon}\right) \\
& =\chi_{i}^{\varepsilon_{j}}\left(\boldsymbol{x}+r_{j} \boldsymbol{z}\right) \Pi\left(\boldsymbol{A}_{i}\left(\epsilon_{\boldsymbol{z}}\left(\boldsymbol{w}^{j}(\boldsymbol{x}, \boldsymbol{z})\right)+\bar{\epsilon}\right)\right),
\end{aligned}
$$

where, for each $\boldsymbol{x}$, in $\Omega$ the function $\boldsymbol{w}^{j}(\boldsymbol{x}, \boldsymbol{z})$ is the $Q$-periodic solution of

$$
-\operatorname{div}_{\boldsymbol{z}}\left(\boldsymbol{C}^{\varepsilon_{j}}\left(\underline{\boldsymbol{A}}, \boldsymbol{x}+r_{j} \boldsymbol{z}\right)\left(\epsilon_{\boldsymbol{z}}\left(\boldsymbol{w}^{j}(\boldsymbol{x}, \boldsymbol{z})\right)+\bar{\epsilon}\right)\right)=0, \quad \text { for } \boldsymbol{z} \text { in } Q .
$$

Here $\boldsymbol{x}$ appears as a parameter and differentiation is carried out in the $\boldsymbol{z}$ variable as indicated by the subscripts. For fixed $\boldsymbol{x}$ the $L^{\infty}(Q)$ norm of

$$
\chi_{i}^{\varepsilon_{j}}\left(\boldsymbol{x}+r_{j} \boldsymbol{z}\right) \Pi\left(\boldsymbol{A}_{i}\left(\epsilon_{\boldsymbol{z}}\left(\boldsymbol{w}^{j}(\boldsymbol{x}, \boldsymbol{z})\right)+\bar{\epsilon}\right)\right)
$$

is computed. This is written as

$$
\left\|\boldsymbol{F}_{j}^{i}(\boldsymbol{x}, \cdot) \boldsymbol{S}^{\mathrm{E}}(\underline{\boldsymbol{A}}, \boldsymbol{x}) \sigma^{\mathrm{M}}(\boldsymbol{x}): \boldsymbol{S}^{\mathrm{E}}(\underline{\boldsymbol{A}}, \boldsymbol{x}) \sigma^{\mathrm{M}}(\boldsymbol{x})\right\|_{L^{\infty}(Q)} .
$$

Denote by $\mathcal{C}$ the class of all sequences $\left\{r_{j}\right\}_{j=1}^{\infty}$ such that

$$
\lim _{j \rightarrow \infty} r_{j}=0 \quad \text { and } \quad \lim _{j \rightarrow \infty} \varepsilon_{j} / r_{j}=0 \text {. }
$$


Set

$$
\begin{aligned}
\tilde{f}^{i}\left(\boldsymbol{x}, \boldsymbol{S}^{\mathrm{E}}(\underline{\boldsymbol{A}}, \boldsymbol{x}) \sigma^{\mathrm{M}}(\boldsymbol{x})\right) \\
\quad=\sup _{\left\{r_{j}\right\}_{j=1}^{\infty} \in \mathcal{C}}\left\{\limsup _{j \rightarrow \infty}\left\|\boldsymbol{F}_{j}^{i}(\boldsymbol{x}, \cdot) \boldsymbol{S}^{\mathrm{E}}(\underline{\boldsymbol{A}}, \boldsymbol{x}) \sigma^{\mathrm{M}}(\boldsymbol{x}): \boldsymbol{S}^{\mathrm{E}}(\underline{\boldsymbol{A}}, \boldsymbol{x}) \sigma^{\mathrm{M}}(\boldsymbol{x})\right\|_{L^{\infty}(Q)}\right\} .
\end{aligned}
$$

The function $\tilde{f}^{i}\left(\boldsymbol{x}, \boldsymbol{S}^{\mathrm{E}}(\underline{\boldsymbol{A}}, \boldsymbol{x}) \sigma^{\mathrm{M}}(\boldsymbol{x})\right)$ is the macrostress modulation function. We now have the following theorem.

Theorem 3.1 (the homogenization constraint). If we consider a sequence $\left\{\boldsymbol{C}^{\varepsilon_{j}}(\underline{\boldsymbol{A}}, \boldsymbol{x})\right\}_{j=1}^{\infty}$ with the associated $2 N+1$-tuple

$$
\left(\boldsymbol{C}^{\mathrm{E}}(\underline{\boldsymbol{A}}, \boldsymbol{x}) ; \theta_{t, 1}(\boldsymbol{x}), \ldots, \theta_{t, N}(\boldsymbol{x}) ; \tilde{f}^{1}\left(\boldsymbol{x}, \boldsymbol{S}^{\mathrm{E}}(\underline{\boldsymbol{A}}, \boldsymbol{x}) \sigma^{\mathrm{M}}(\boldsymbol{x})\right), \ldots, \tilde{f}^{N}\left(\boldsymbol{x}, \boldsymbol{S}^{\mathrm{E}}(\underline{\boldsymbol{A}}, \boldsymbol{x}) \sigma^{\mathrm{M}}(\boldsymbol{x})\right)\right),
$$

then we have

$$
\theta_{t, i}(\boldsymbol{x})\left(\tilde{f}^{i}\left(\boldsymbol{x}, \boldsymbol{S}^{\mathrm{E}}(\underline{\boldsymbol{A}}, \boldsymbol{x}) \sigma^{\mathrm{M}}(\boldsymbol{x})\right)-t\right) \geqslant 0, \quad i=1, \ldots, N,
$$

almost everywhere on $\Omega$.

The next corollary is an immediate consequence of theorem 3.1.

Corollary 3.2. Given the hypothesis of theorem 3.1, suppose that

$$
t>\tilde{f}^{i}\left(\boldsymbol{x}, \boldsymbol{S}^{\mathrm{E}}(\underline{\boldsymbol{A}}, \boldsymbol{x}) \sigma^{\mathrm{M}}(\boldsymbol{x})\right)
$$

on a set $\omega$, where $\omega \subset \Omega$. Then the stress in the $i$ th phase satisfies $\Pi\left(\sigma^{\varepsilon_{j}}\right) \leqslant t$ almost everywhere on $\omega$ outside $S_{t, i}^{\varepsilon_{j}} \cap \omega$, moreover the measure of the set $S_{t, i}^{\varepsilon_{j}} \cap \omega$ tends to zero in the fine-scale limit.

We denote the set of points in $\Omega$ where $\Pi\left(\sigma^{\varepsilon_{j}}\right)>t$ by $S_{t}^{\varepsilon_{j}}$, and one has the following corollary.

Corollary 3.3. Given the hypothesis of theorem 3.1, suppose that

$$
t>\tilde{f}^{i}\left(\boldsymbol{x}, \boldsymbol{S}^{\mathrm{E}}(\underline{\boldsymbol{A}}, \boldsymbol{x}) \sigma^{\mathrm{M}}(\boldsymbol{x})\right)
$$

for $i=1, \ldots, N$ on a set $\omega$, where $\omega \subset \Omega$. Then $\Pi\left(\sigma^{\varepsilon_{j}}\right) \leqslant t$ almost everywhere on $\omega$ outside $S_{t}^{\varepsilon_{j}} \cap \omega$, moreover the measure of the set $S_{t}^{\varepsilon_{j}} \cap \omega$ tends to zero in the fine-scale limit.

We demonstrate that a pointwise bound on the macrostress modulation function delivers a pointwise bound on the equivalent stress in the composite when the microstructure is sufficiently fine relative to the size of the domain and variation of the body force.

Corollary 3.4 (pointwise bounds on the equivalent stress). Given the hypothesis of theorem 3.1, suppose that

$$
t>\tilde{f}^{i}\left(\boldsymbol{x}, \boldsymbol{S}^{\mathrm{E}}(\underline{\boldsymbol{A}}, \boldsymbol{x}) \sigma^{\mathrm{M}}(\boldsymbol{x})\right)
$$

for $i=1, \ldots, N$ on a set $\omega$, where $\omega \subset \Omega$. One can then pass to a subsequence if necessary to find that there is a critical $\varepsilon_{0}$ such that, for every $\varepsilon_{j}<\varepsilon_{0}$,

$$
\Pi\left(\sigma^{\varepsilon_{j}}(\boldsymbol{x})\right) \leqslant t
$$

for almost every $\boldsymbol{x}$ in $\omega$. Here $\varepsilon_{0}$ can depend upon position. 
Set

$$
\tilde{t}=\sup _{\boldsymbol{x} \in \omega}\left(\max _{i}\left\{\tilde{f}^{i}\left(\boldsymbol{x}, \boldsymbol{S}^{\mathrm{E}}(\underline{\boldsymbol{A}}, \boldsymbol{x}) \sigma^{\mathrm{M}}(\boldsymbol{x})\right)\right\}\right) .
$$

From corollary 3.4 one then has a critical scale $\varepsilon_{0}$ (depending possibly on $\boldsymbol{x}$ ) such that, for every $\varepsilon_{j}<\varepsilon_{0}$,

$$
\Pi\left(\sigma^{\varepsilon_{j}}(\boldsymbol{x})\right) \leqslant \tilde{t}
$$

for almost all points in $\omega$.

Proof of corollary 3.4. Since $\lim _{j \rightarrow \infty}\left|S_{t}^{\varepsilon_{j}} \cap \omega\right|=0$, we can choose

$$
\left\{\boldsymbol{C}^{\varepsilon_{j_{k}}}(\underline{\boldsymbol{A}}, \boldsymbol{x},)\right\}_{k=1}^{\infty}
$$

with stresses $\left\{\sigma^{\varepsilon_{j_{k}}}\right\}_{k=1}^{\infty}$ that satisfy $\left|S_{t}^{\varepsilon_{j_{k}}} \cap \omega\right|<2^{-k}$. Then if $\boldsymbol{x}$ does not belong to $\bigcup_{k \geqslant \tilde{K}}^{\infty} S_{t}^{\varepsilon_{j_{k}}} \cap \omega$, one has that $\Pi\left(\sigma^{\varepsilon_{j_{k}}}\right) \leqslant t$ for every $k>\tilde{K}$. Hence, for any $\boldsymbol{x}$ not in $A=\bigcap_{K=1}^{\infty} \bigcup_{k \geqslant K}^{\infty} S_{t}^{\varepsilon_{j_{k}}} \cap \omega$ there is an index $K$ for which $\Pi\left(\sigma^{\varepsilon_{j_{k}}}\right) \leqslant t$ for every $k>K$. But

$$
|A| \leqslant\left|\bigcup_{k \geqslant \tilde{K}}^{\infty} S_{t}^{\varepsilon_{j_{k}}} \cap \omega\right| \leqslant \sum_{k=\tilde{K}}^{\infty}\left|S_{t}^{\varepsilon_{j_{k}}} \cap \omega\right| \leqslant 2^{-\tilde{K}+1} .
$$

Hence $|A|=0$. Thus for almost every $\boldsymbol{x}$ in $\omega$ there is a finite index $K$ (that may depend upon $\boldsymbol{x}$ ) for which $\Pi\left(\sigma^{\varepsilon_{j_{k}}}\right) \leqslant t$ for every $k>K$ and the corollary follows.

Conversely, it follows from (3.8) that

$$
\tilde{f}^{i}\left(\boldsymbol{x}, \boldsymbol{S}^{\mathrm{E}}(\underline{\boldsymbol{A}}, \boldsymbol{x}) \sigma^{\mathrm{M}}(\boldsymbol{x})\right) \geqslant t,
$$

on sets where $\theta_{t, i}(\boldsymbol{x})>0$. It is evident that $\tilde{f}^{i}\left(\boldsymbol{x}, \boldsymbol{S}^{\mathrm{E}}(\underline{\boldsymbol{A}}, \boldsymbol{x}) \sigma^{\mathrm{M}}(\boldsymbol{x})\right)=\infty$ on sets $\omega$ for which $\theta_{t, i}>0$ for every $t>0$.

Next we provide conditions for which upper bounds on $\lim \sup _{j \rightarrow \infty}\left\|\chi_{i}^{\epsilon_{j}} \Pi\left(\sigma^{\epsilon_{j}}\right)\right\|_{\infty}$ can be obtained in terms of homogenized quantities.

Corollary 3.5. Suppose it is known that $\ell=\lim \sup _{j \rightarrow \infty}\left\|\chi_{i}^{\epsilon_{j}} \Pi\left(\sigma^{\epsilon_{j}}\right)\right\|_{\infty}<\infty$. Suppose further that for an interval $0<\delta<b$ the measure (volume) of the sets where $\theta_{\ell-\delta, i}>0$ does not vanish. Then

$$
\limsup _{j \rightarrow \infty}\left\|\chi_{i}^{\epsilon_{j}} \Pi\left(\sigma^{\epsilon_{j}}\right)\right\|_{\infty} \leqslant\left\|\tilde{f}^{i}\left(\boldsymbol{x}, \boldsymbol{S}^{\mathrm{E}}(\underline{\boldsymbol{A}}, \boldsymbol{x}) \sigma^{\mathrm{M}}(\boldsymbol{x})\right)\right\|_{\infty}
$$

Corollary 3.5 follows upon setting $t=\ell-\delta$ and applying (3.14).

Next, bounds are obtained on $\limsup _{j \rightarrow \infty}\left\|\chi_{i}^{\epsilon_{j}} \Pi\left(\sigma^{\epsilon_{j}}\right)\right\|_{p}$ for $1 \leqslant p<\infty$.

Corollary 3.6. Suppose that $\limsup _{j \rightarrow \infty}\left\|\chi_{i}^{\epsilon_{j}} \Pi\left(\sigma^{\epsilon_{j}}\right)\right\|_{\infty}<\infty$. Then

$$
\limsup _{j \rightarrow \infty}\left\|\chi_{i}^{\epsilon_{j}} \Pi\left(\sigma^{\epsilon_{j}}\right)\right\|_{p} \leqslant\left\|\tilde{f}^{i}\left(\boldsymbol{x}, \boldsymbol{S}^{\mathrm{E}}(\underline{\boldsymbol{A}}, \boldsymbol{x}) \sigma^{\mathrm{M}}(\boldsymbol{x})\right)\right\|_{\infty}|\Omega|^{1 / p},
$$

for any $p, 1 \leqslant p<\infty$.

Proof of corollary 3.6. For $t \geqslant 0$ we consider the set of points $S_{t, i .}^{\varepsilon_{j}}$ in the $i$ th phase where $\Pi\left(\sigma^{\varepsilon_{j}}\right)>t$. The distribution function $\lambda_{i}^{j}(t)$ is defined by $\lambda_{i}^{j}(t)=\left|S_{t, i}^{\varepsilon_{j}}\right|$. For each $j$ the distribution function is non-increasing continuous from the right and 
therefore measurable. Set $\ell=\limsup _{j \rightarrow \infty}\left\|\chi_{i}^{\epsilon_{j}} \Pi\left(\sigma^{\epsilon_{j}}\right)\right\|_{\infty}$. Choose $r>0$, then from the hypotheses $\lambda_{i}^{j}$ has support in $[0, \ell+r)$ for sufficiently large $j$. For each $j$ one has

$$
\int_{\Omega} \chi_{i}^{\varepsilon_{j}}\left(\Pi\left(\sigma^{\epsilon_{j}}\right)\right)^{p} \mathrm{~d} \boldsymbol{x}=p \int_{0}^{\infty} t^{p-1} \lambda_{i}^{j}(t) \mathrm{d} t .
$$

It is easy to see that the function $g_{p}(t)$ defined by $g_{p}(t)=t^{p-1}|\Omega|$ for $0 \leqslant t<\ell+r$ and $g_{p}(t)=0$ for $\ell+r \leqslant t<\infty$ is integrable on $[0, \infty)$ and $t^{p-1} \lambda_{i}^{j}(t) \leqslant g_{p}(t)$ for sufficiently large $j$. Thus an application of Lebesgue's theorem gives

$$
\limsup _{j \rightarrow \infty} \int_{\Omega} \chi_{i}^{\varepsilon_{j}}\left(\Pi\left(\sigma^{\epsilon_{j}}\right)\right)^{p} \mathrm{~d} \boldsymbol{x} \leqslant p \int_{0}^{\infty} t^{p-1} \limsup _{j \rightarrow \infty}\left(\lambda_{i}^{j}(t)\right) \mathrm{d} t .
$$

For each $t$ we pass to a subsequence if necessary and appeal to (3.3) to obtain a density $\theta_{t, i}(\boldsymbol{x})$ for which

$$
\limsup _{j \rightarrow \infty} \lambda_{i}^{j}(t)=\int_{\Omega} \theta_{t, i}(\boldsymbol{x}) \mathrm{d} \boldsymbol{x},
$$

with $0 \leqslant \theta_{t, i}(\boldsymbol{x}) \leqslant 1$. It follows from theorem 3.1 that $\theta_{t, i}(\boldsymbol{x})=0$ almost everywhere if $t>\tilde{\mathcal{J}}=\left\|\tilde{f}^{i}\left(\boldsymbol{x}, \boldsymbol{S}^{\mathrm{E}}(\underline{\boldsymbol{A}}, \boldsymbol{x}) \sigma^{\mathrm{M}}(\boldsymbol{x})\right)\right\|_{\infty}$. It is now evident that

$$
\limsup _{j \rightarrow \infty} \int_{\Omega} \chi_{i}^{\varepsilon_{j}}\left(\Pi\left(\sigma^{\epsilon_{j}}\right)\right)^{p} \mathrm{~d} \boldsymbol{x} \leqslant p \int_{0}^{\tilde{\mathcal{J}}} t^{p-1} \int_{\Omega} \theta_{t, i}(\boldsymbol{x}) \mathrm{d} \boldsymbol{x} \mathrm{d} t .
$$

The corollary follows from the estimate $|\Omega| \geqslant \int_{\Omega} \theta_{t, i}(\boldsymbol{x}) \mathrm{d} \boldsymbol{x}$.

\section{(a) Proof of theorem 3.1}

Consider the set of points $S_{t, i}^{\varepsilon_{j}}$ in the $i$ th phase for which $\Pi\left(\sigma^{\varepsilon_{j}}\right)>t$ for a fixed positive number $t$. The characteristic function for $S_{t, i}^{\varepsilon_{j}}$ is denoted by $\chi_{t, i}^{j}(\boldsymbol{x})$. Passing to a subsequence if necessary one has that $\chi_{t, i}^{j} \stackrel{*}{\rightarrow} \theta_{t, i}$ in $L^{\infty}(\Omega)$ as $j \rightarrow \infty$. Here the density $\theta_{t, i}(\boldsymbol{x})$ takes values in $[0,1]$. For any non-negative smooth test function $p(\boldsymbol{x})$ one has

$$
t \int_{\Omega} p \chi_{t, i}^{j} \mathrm{~d} \boldsymbol{x} \leqslant \int_{\Omega} p \chi_{t, i}^{j} \Pi\left(\sigma^{\varepsilon_{j}}\right) \mathrm{d} \boldsymbol{x} .
$$

Taking the limit on the left-hand side of (3.19) gives $t \int_{\Omega} p \theta_{t, i} \mathrm{~d} \boldsymbol{x}$. The goal is to pass to the limit on the right-hand side of (3.19) to obtain a pointwise upper bound on the product $t \theta_{t, i}$. This will follow from the following lemma.

Lemma 3.7. There exists a subsequence of $\left\{\chi_{t, i}^{j}\right\}_{j=1}^{\infty}$ and $\left\{\sigma^{\varepsilon_{j}}\right\}_{j=1}^{\infty}$ also denoted by $\left\{\chi_{t, i}^{j}\right\}_{j=1}^{\infty}$ and $\left\{\sigma^{\varepsilon_{j}}\right\}_{j=1}^{\infty}$ such that

$$
\chi_{t, i}^{j} \Pi\left(\sigma^{\varepsilon_{j}}\right) \rightarrow \boldsymbol{A}_{i} \boldsymbol{\Pi} \boldsymbol{A}_{i} \boldsymbol{H}_{t}(\boldsymbol{x}) \boldsymbol{S}^{\mathrm{E}}(\underline{\boldsymbol{A}}, \boldsymbol{x}) \sigma^{\mathrm{M}}(\boldsymbol{x}): \boldsymbol{S}^{\mathrm{E}}(\underline{\boldsymbol{A}}, \boldsymbol{x}) \sigma^{\mathrm{M}}(\boldsymbol{x})
$$

in the sense of distributions. Here $\boldsymbol{A}_{i} \boldsymbol{\Pi} \boldsymbol{A}_{i} \boldsymbol{H}_{t}(\boldsymbol{x})$ is given by

$\boldsymbol{A}_{i} \boldsymbol{\Pi} \boldsymbol{A}_{i} \boldsymbol{H}_{t}(\boldsymbol{x}) \bar{\epsilon}: \bar{\epsilon}$

$$
\begin{aligned}
& =\lim _{r \rightarrow 0} \lim _{j \rightarrow \infty}\left(\frac{1}{|Q(\boldsymbol{x}, r)|}\right) \int_{Q(\boldsymbol{x}, r)} \chi_{t, i}^{j} \boldsymbol{\Pi}\left(\boldsymbol{A}_{i}\left(\epsilon\left(\boldsymbol{w}_{\bar{\epsilon}}^{j, r}\right)+\bar{\epsilon}\right)\right):\left(\boldsymbol{A}_{i}\left(\epsilon\left(\boldsymbol{w}_{\bar{\epsilon}}^{j, r}\right)+\bar{\epsilon}\right)\right) \mathrm{d} \boldsymbol{y} \\
& =\lim _{r \rightarrow 0} \lim _{j \rightarrow \infty}\left(\frac{1}{|Q(\boldsymbol{x}, r)|}\right) \int_{Q(\boldsymbol{x}, r)} \chi_{t, i}^{j} \Pi\left(\boldsymbol{A}_{i}\left(\epsilon\left(\boldsymbol{w}_{\bar{\epsilon}}^{j, r}\right)+\bar{\epsilon}\right)\right) \mathrm{d} \boldsymbol{y}
\end{aligned}
$$


where $\bar{\epsilon}$ is any constant strain and the local oscillatory response function is denoted by $\boldsymbol{w}_{\bar{\epsilon}}^{j, r}$. Here $\boldsymbol{w}_{\bar{\epsilon}}^{j, r}$ is the $Q(\boldsymbol{x}, r)$-periodic solution of

$$
-\operatorname{div}\left(\boldsymbol{C}^{\varepsilon_{j}}(\underline{\boldsymbol{A}}, \boldsymbol{y})\left(\epsilon\left(\boldsymbol{w}_{\bar{\epsilon}}^{j, r}(\boldsymbol{y})\right)+\bar{\epsilon}\right)\right)=0, \quad \text { for } \boldsymbol{y} \text { in } Q(\boldsymbol{x}, r) .
$$

Proof of lemma 3.7. One introduces a sequence of $N+1$ phase composites with the following properties: on the set $S_{t, i}^{\varepsilon_{j}}$ the elasticity is $\boldsymbol{P}_{N+1}$; for points in the $i$ th phase not in $S_{t, i}^{\varepsilon_{j}}$ the elasticity is $\boldsymbol{P}_{i}$; in the other phases the elasticity is $\boldsymbol{P}_{m}$ in the $m$ th phase $m=1, \ldots, N$, for $m \neq i$. We denote the elastic tensor for this composite by $\boldsymbol{C}^{\varepsilon_{j}}\left(\underline{\boldsymbol{P}}, \boldsymbol{P}_{N+1}, \boldsymbol{x}\right)$. This tensor is defined in terms of characteristic functions by

$$
\boldsymbol{C}^{\varepsilon_{j}}\left(\underline{\boldsymbol{P}}, \boldsymbol{P}_{N+1}, \boldsymbol{x}\right)=\sum_{\substack{m=1, m \neq i}}^{N} \chi_{m}^{\varepsilon_{j}}(\boldsymbol{x}) \boldsymbol{P}_{m}+\left(\chi_{i}^{\varepsilon_{j}}(\boldsymbol{x})-\chi_{t, i}^{j}(\boldsymbol{x})\right) \boldsymbol{P}_{i}+\chi_{t, i}^{j}(\boldsymbol{x}) \boldsymbol{P}_{N+1} .
$$

Most importantly, we observe that $\boldsymbol{C}^{\varepsilon_{j}}(\underline{\boldsymbol{A}}, \boldsymbol{x})=\boldsymbol{C}^{\varepsilon_{j}}\left(\underline{\boldsymbol{A}}, \boldsymbol{A}_{i}, \boldsymbol{x}\right)$. Passing to subsequences as necessary, the sequence

$$
\left\{\boldsymbol{C}^{\varepsilon_{j}}\left(\underline{\boldsymbol{P}}, \boldsymbol{P}_{N+1}, \boldsymbol{x}\right)\right\}_{j=1}^{\infty}
$$

G-converges to $\boldsymbol{C}^{\mathrm{E}}\left(\underline{\boldsymbol{P}}, \boldsymbol{P}_{N+1}, \boldsymbol{x}\right)$ for every $\left(\underline{\boldsymbol{P}}, \boldsymbol{P}_{N+1}\right)$ in a neighbourhood of $\left(\underline{\boldsymbol{A}}, \boldsymbol{A}_{i}\right)$. Observing that $\boldsymbol{A}_{i} \boldsymbol{\Pi} \boldsymbol{A}_{i} \boldsymbol{H}_{t}(\boldsymbol{x})=\boldsymbol{A}_{i} \boldsymbol{\Pi} \boldsymbol{A}_{i} \nabla^{N+1} \boldsymbol{C}^{\mathrm{E}}\left(\underline{\boldsymbol{A}}, \boldsymbol{A}_{i}, \boldsymbol{x}\right)$, the lemma then follows from the same arguments as those used to prove theorem 2.3.

We pass to subsequences according to lemma 3.7, and on taking limits in (3.19) it follows that

$$
\boldsymbol{A}_{i} \boldsymbol{\Pi} \boldsymbol{A}_{i} \boldsymbol{H}_{t}(\boldsymbol{x}) \boldsymbol{S}^{\mathrm{E}}(\underline{\boldsymbol{A}}, \boldsymbol{x}) \sigma^{\mathrm{M}}(\boldsymbol{x}): \boldsymbol{S}^{\mathrm{E}}(\underline{\boldsymbol{A}}, \boldsymbol{x}) \sigma^{\mathrm{M}}(\boldsymbol{x})-t \theta_{t, i}(\boldsymbol{x}) \geqslant 0,
$$

almost everywhere on $\Omega$. Passing to a diagonal sequence we have $\lim _{j \rightarrow \infty} r_{j}=0$, $\lim _{j \rightarrow \infty} \varepsilon_{j} / r_{j}=0$

$$
\boldsymbol{A}_{i} \boldsymbol{\Pi} \boldsymbol{A}_{i} \boldsymbol{H}_{t}(\boldsymbol{x}) \bar{\epsilon}: \bar{\epsilon}=\lim _{j \rightarrow \infty}\left(\frac{1}{\left|Q\left(\boldsymbol{x}, r_{j}\right)\right|}\right) \int_{Q\left(\boldsymbol{x}, r_{j}\right)} \chi_{t, i}^{j} \Pi\left(\boldsymbol{A}_{i}\left(\epsilon\left(\boldsymbol{w}_{\bar{\epsilon}}^{j, r_{j}}\right)+\bar{\epsilon}\right)\right) \mathrm{d} \boldsymbol{y}
$$

and at Lebesgue points of $\theta_{t, i}$

$$
\lim _{j \rightarrow \infty}\left(\frac{1}{\left|Q\left(\boldsymbol{x}, r_{j}\right)\right|}\right) \int_{Q\left(\boldsymbol{x}, r_{j}\right)} \chi_{t, i}^{j} \mathrm{~d} \boldsymbol{x}=\theta_{t, i}(\boldsymbol{x}) .
$$

Here $\boldsymbol{w}_{\bar{\epsilon}}^{j, r_{j}}$ solves (3.21) with $r=r_{j}$. Now change coordinates by translation and re-scaling so that $Q\left(\boldsymbol{x}, r_{j}\right)$ is transformed to the unit cube $Q$ centred at the origin. The change of variables is given by $\boldsymbol{y}=\boldsymbol{x}+\boldsymbol{z} r_{j}$, where $\boldsymbol{z}$ lies in $Q$. One writes $\boldsymbol{w}_{\bar{\epsilon}}^{j, r_{j}}(\boldsymbol{y})=r_{j} \phi^{j}\left((\boldsymbol{y}-\boldsymbol{x}) / r_{j}\right)$, where $\phi^{j}(\boldsymbol{z})$ is the $Q$-periodic solution of

$$
-\operatorname{div}\left(\boldsymbol{C}^{\varepsilon_{j}}\left(\underline{\boldsymbol{A}}, \boldsymbol{x}+r_{j} \boldsymbol{z}\right)\left(\epsilon\left(\phi^{j}(\boldsymbol{z})\right)+\bar{\epsilon}\right)\right)=0, \text { for } \boldsymbol{z} \text { in } Q .
$$

Under this change of coordinates $\chi_{t, i}^{j}=\chi_{t, i}^{j}\left(\boldsymbol{x}+r_{j} \boldsymbol{z}\right)$ and

$$
\boldsymbol{A}_{i} \boldsymbol{\Pi} \boldsymbol{A}_{i} \boldsymbol{H}_{t}(\boldsymbol{x}) \bar{\epsilon}: \bar{\epsilon}=\lim _{j \rightarrow \infty} \int_{Q} \chi_{t, i}^{j}\left(\boldsymbol{x}+r_{j} \boldsymbol{z}\right) \Pi\left(\boldsymbol{A}_{i}\left(\epsilon\left(\phi^{j}(\boldsymbol{z})\right)+\bar{\epsilon}\right)\right) \mathrm{d} \boldsymbol{z} .
$$


Application of Hölder's inequality gives

$$
\begin{aligned}
\boldsymbol{A}_{i} \boldsymbol{\Pi} \boldsymbol{A}_{i} & \boldsymbol{H}_{t}(\boldsymbol{x}) \bar{\epsilon}: \bar{\epsilon} \\
& \leqslant\left(\limsup _{j \rightarrow \infty}\left(\frac{1}{|Q|}\right) \int_{Q} \chi_{t, i}^{j}\left(\boldsymbol{x}+r_{j} \boldsymbol{z}\right) \mathrm{d} \boldsymbol{z}\right) \limsup _{j \rightarrow \infty}\left\|\boldsymbol{F}_{j}^{i}(\boldsymbol{x}, \cdot) \bar{\epsilon}: \bar{\epsilon}\right\|_{L^{\infty}(Q)} \\
& =\theta_{t, i}(\boldsymbol{x}) \limsup _{j \rightarrow \infty}\left\|\boldsymbol{F}_{j}^{i}(\boldsymbol{x}, \cdot) \bar{\epsilon}: \bar{\epsilon}\right\|_{L^{\infty}(Q)} .
\end{aligned}
$$

Theorem 3.1 now follows from (3.22) and noting that the upper bound (3.27) holds for a subsequence of $\left\{\chi_{i}^{\varepsilon_{j}}\right\}_{j=1}^{\infty}$ and for a particular choice of $\left\{r_{j}\right\}_{j=1}^{\infty}$ in $\mathcal{C}$.

\section{A homogenized constraint condition and bounds on stress for piecewise constant and continuously graded microstructures}

In this section we consider microstructures that are piecewise constant or change continuously across the structure. Here the microstructure is assumed to be locally periodic. A graded locally periodic composite requires a description at both macroscopic and microscopic length-scales. We start by describing the periodic microstructure. Periodic configurations of $N$ elastic phases in the unit cube $Q$ are considered. Here $Q$ is the unit cell for the periodic microstructure; however, more general period cells could be used. The elasticity tensor of each phase is specified by $\boldsymbol{A}_{i}$, for $i=1, \ldots, N$. A particular choice of component elasticity tensors is specified by the array $\underline{\boldsymbol{A}}=\left(\boldsymbol{A}_{1}, \boldsymbol{A}_{2}, \boldsymbol{A}_{3}, \ldots, \boldsymbol{A}_{N}\right)$. The elasticity tensor for a given configuration in the unit cube is written $\boldsymbol{C}_{0}(\underline{\boldsymbol{A}}, \boldsymbol{y})$. Here $\boldsymbol{C}_{0}(\underline{\boldsymbol{A}}, \boldsymbol{y})=\boldsymbol{A}_{i}$ for $\boldsymbol{y}$ in the $i$ th phase and is periodic in $\boldsymbol{y}$.

A periodic microstructure occupying $\Omega$ of length-scale $1 / \nu$ is described by the simple re-scaling $\boldsymbol{C}_{0}(\underline{\boldsymbol{A}}, \nu \boldsymbol{x})$. Here the periodic microgeometry remains the same as $\boldsymbol{x}$ traverses $\Omega$. To describe the spatial variation of the microstructure across $\Omega$, it is convenient to start with a function $\boldsymbol{C}_{0}(\underline{\boldsymbol{A}}, \boldsymbol{x}, \boldsymbol{y})$ defined on $\Omega \times Q$. For each $\boldsymbol{x}$, the function $\boldsymbol{C}_{0}(\underline{\boldsymbol{A}}, \boldsymbol{x}, \cdot)$ describes an elasticity tensor for a configuration in the unit period cell $Q$. We consider two types of graded locally periodic composites. The first locally periodic microstructure corresponds to periodic microgeometries that are constant on fixed subdomains of $\Omega$. The structural domain $\Omega$ is partitioned into subdomains $O_{\ell}^{\kappa}$ of diameter less than or equal to $\kappa$. Here $\Omega=\bigcup_{\ell} O_{\ell}^{\kappa}$. Associated with each set $O_{\ell}^{\kappa}$ we consider a function $\boldsymbol{C}_{0}^{\ell}(\underline{\boldsymbol{A}}, \boldsymbol{y})$. The piecewise constant locally periodic microgeometry is given in terms of the function

$$
\boldsymbol{C}_{0}(\underline{\boldsymbol{A}}, \boldsymbol{x}, \boldsymbol{y})=\boldsymbol{C}_{0}^{\ell}(\underline{\boldsymbol{A}}, \boldsymbol{y})
$$

when $\boldsymbol{x}$ lies in $O_{\ell}^{\kappa}$. For $\nu=1,2, \ldots$ the locally periodic microstructure is given by $\boldsymbol{C}_{0}(\underline{\boldsymbol{A}}, \boldsymbol{x}, \boldsymbol{x} \nu)$. Here the microgeometry is the same inside each $O_{\ell}^{\kappa}$ but can be different for each $O_{\ell}^{\kappa}$. The fine-scale limit for the family of composites is obtained by sending $1 / \nu$ to zero. This type of graded microstructure is considered in Bensoussan et al. (1978). The effective elastic property obtained in the fine-scale limit is derived in Bensoussan et al. (1978, ch. 6). Here the effective elastic tensor is a function of position taking different values on each set $O_{\ell}^{\kappa}$. This type of microgeometry is referred to in this article as a piecewise constant locally periodic microstructure.

The second type of graded composite admits a continuous variation of microscopic properties. One considers functions $\boldsymbol{C}_{0}(\underline{\boldsymbol{A}}, \boldsymbol{x}, \boldsymbol{y})$ that are continuous in the $\boldsymbol{x}$ variable. 
Here the continuity is specified by

$$
\lim _{\boldsymbol{h} \rightarrow 0} \int_{Q}\left|\boldsymbol{C}_{0}(\underline{\boldsymbol{A}}, \boldsymbol{x}+\boldsymbol{h}, \boldsymbol{y})-\boldsymbol{C}_{0}(\underline{\boldsymbol{A}}, \boldsymbol{x}, \boldsymbol{y})\right| \mathrm{d} \boldsymbol{y}=0 .
$$

As before, the structural domain $\Omega$ is partitioned into subdomains $O_{\ell}^{\kappa}$ of diameter less than or equal to $\kappa$. The locally periodic microgeometry is given in terms of the function denoted by $\boldsymbol{C}_{0}^{\kappa}(\underline{\boldsymbol{A}}, \boldsymbol{x}, \boldsymbol{y})$ and is defined in the following way: in the $\ell$ th subdomain $\boldsymbol{C}_{0}^{\kappa}(\underline{\boldsymbol{A}}, \boldsymbol{x}, \boldsymbol{y})=\boldsymbol{C}_{0}\left(\underline{\boldsymbol{A}}, \boldsymbol{x}^{\ell}, \boldsymbol{y}\right)$, where $\boldsymbol{x}^{\ell}$ is a sample point chosen from $O_{\ell}^{\kappa}$. The locally periodic microstructure is described by the elasticity tensor $\boldsymbol{C}_{0}^{\kappa}(\underline{\boldsymbol{A}}, \boldsymbol{x}, \nu \boldsymbol{x})$. The continuity condition (4.2) ensures that near by subdomains have nearly the same microgeometies. This type of microstructure is referred to as a continuously graded locally periodic microstructure. In this context the fine-scale limit is obtained by considering a family of partitions indexed by $j=1,2 \ldots$, with subdomains $O_{\ell}^{\kappa_{j}}$ of diameter less than or equal to $\kappa_{j}$. Here $\kappa_{j}$ goes to zero as $j$ goes to infinity and the scale of the microstructure is given by $1 / \nu_{j}$, where $1 / \nu_{j}$ goes to zero as $j$ tends to infinity and $\left(1 / \nu_{j}\right) / \kappa_{j} \rightarrow 0$. The associated sequence of elasticity tensors is written as $\left\{\boldsymbol{C}_{0}^{\kappa_{j}}\left(\underline{\boldsymbol{A}}, \boldsymbol{x}, \boldsymbol{x} \nu_{j}\right)\right\}_{j=1}^{\infty}$.

To expedite the presentation we introduce the functions $\chi_{i}(\boldsymbol{x}, \boldsymbol{y}), i=1, \ldots, N$, defined on $\Omega \times Q$ such that for each $\boldsymbol{x}$ the function $\chi_{i}(\boldsymbol{x}, \cdot)$ is the characteristic function of the $i$ th phase in the unit period cell $Q$. For $\boldsymbol{x}$ fixed, $\chi_{i}(\boldsymbol{x}, \boldsymbol{y})=1$ when $\boldsymbol{y}$ is in the $i$ th phase and zero otherwise and we write

$$
\boldsymbol{C}_{0}(\underline{\boldsymbol{A}}, \boldsymbol{x}, \boldsymbol{y})=\sum_{i}^{N} \chi_{i}(\boldsymbol{x}, \boldsymbol{y}) \boldsymbol{A}_{i}
$$

The effective elastic tensor is defined by

$$
\boldsymbol{C}^{\mathrm{E}}(\underline{\boldsymbol{A}}, \boldsymbol{x}) \bar{\epsilon}: \bar{\epsilon}=\int_{Q} \boldsymbol{C}_{0}(\underline{\boldsymbol{A}}, \boldsymbol{x}, \boldsymbol{y})(\epsilon(\boldsymbol{w})+\bar{\epsilon}):(\epsilon(\boldsymbol{w})+\bar{\epsilon}) \mathrm{d} \boldsymbol{y},
$$

where for each fixed value of $\boldsymbol{x}$, the function $\boldsymbol{w}(\boldsymbol{x}, \boldsymbol{y})$ is the $Q$-periodic solution of

$$
-\operatorname{div}_{\boldsymbol{y}}\left(\boldsymbol{C}_{0}(\underline{\boldsymbol{A}}, \boldsymbol{x}, \boldsymbol{y})\left(\epsilon_{\boldsymbol{y}}(\boldsymbol{w}(\boldsymbol{x}, \boldsymbol{y}))+\bar{\epsilon}\right)\right)=0 .
$$

Here $\boldsymbol{x}$ appears as a parameter and the subscripts indicate differentiation with respect to the $\boldsymbol{y}$ variable.

The effective property associated with the fine-scale limit of the piecewise constant periodic microstructure is given by (4.4) with $\boldsymbol{C}_{0}(\underline{\boldsymbol{A}}, \boldsymbol{x}, \boldsymbol{y})$ given by $(4.1)$; this is proved in Bensoussan et al. (1978).

Next we consider continuously graded locally periodic microstructures. The homogenization limit of the elasticity tensors $\left\{\boldsymbol{C}_{0}^{\kappa_{j}}\left(\underline{\boldsymbol{A}}, \boldsymbol{x}, \boldsymbol{x} \nu_{j}\right)\right\}_{j=1}^{\infty}$ is given by the following theorem.

Theorem 4.1. The sequence of local elasticity tensors $\left\{\boldsymbol{C}_{0}^{\kappa_{j}}\left(\underline{\boldsymbol{A}}, \boldsymbol{x}, \boldsymbol{x} \nu_{j}\right)\right\}_{j=1}^{\infty}$ is a $G$-convergent sequence, and its $G$-limit is the effective tensor $\boldsymbol{C}^{\mathrm{E}}(\underline{\boldsymbol{A}}, \boldsymbol{x})$ defined by (4.4). Conversely, for any effective tensor $\boldsymbol{C}^{\mathrm{E}}(\underline{\boldsymbol{A}}, \boldsymbol{x})$ given by (4.4), where $\boldsymbol{C}_{0}(\underline{\boldsymbol{A}}, \boldsymbol{x}, \boldsymbol{y})$ is given by (4.3) and satisfies (4.2), one can construct a sequence $\left\{\boldsymbol{C}_{0}^{\kappa_{j}}\left(\underline{\boldsymbol{A}}, \boldsymbol{x}, \boldsymbol{x} \nu_{j}\right)\right\}_{j=1}^{\infty}$ that G-converges to it. The effective tensor is a continuous function of position. 
In what follows, we consider the fine-scale limits of piecewise constant locally periodic microstructures and continuously graded locally periodic microstructures. Because there are more technicalities involved for continuously graded locally periodic microstructures, we state and prove the following theorems for this case and note that the same holds for the piecewise constant locally periodic microstructures.

We prescribe a function of the form $\boldsymbol{C}_{0}(\underline{\boldsymbol{A}}, \boldsymbol{x}, \boldsymbol{y})$ given by (4.3) and satisfying (4.2). We consider the associated sequence of elasticity tensors $\left\{\boldsymbol{C}_{0}^{\kappa_{j}}\left(\underline{\boldsymbol{A}}, \boldsymbol{x}, \boldsymbol{x} \nu_{j}\right)\right\}_{j=1}^{\infty}$ corresponding to a sequence of continuously graded microstructures. For a prescribed body load $\boldsymbol{f}$ the elastic displacements $\boldsymbol{u}^{\nu_{j}}$ are solutions of

$$
-\operatorname{div}\left(\boldsymbol{C}_{0}^{\kappa_{j}}\left(\underline{\boldsymbol{A}}, \boldsymbol{x}, \boldsymbol{x} \nu_{j}\right) \epsilon\left(\boldsymbol{u}^{\nu_{j}}(\boldsymbol{x})\right)\right)=\boldsymbol{f},
$$

with $\boldsymbol{u}^{\nu_{j}}=0$ on the boundary of $\Omega$. The associated stresses are given by $\sigma^{\nu_{j}}=$ $\boldsymbol{C}_{0}^{\kappa_{j}}\left(\underline{\boldsymbol{A}}, \boldsymbol{x}, \boldsymbol{x} \nu_{j}\right) \epsilon\left(\boldsymbol{u}^{\nu_{j}}(\boldsymbol{x})\right)$.

From theorem 4.1 the sequence $\left\{\boldsymbol{C}_{0}^{\kappa_{j}}\left(\underline{\boldsymbol{A}}, \boldsymbol{x}, \boldsymbol{x} \nu_{j}\right)\right\}_{j=1}^{\infty}$ G-converges to the effective elasticity tensor $\boldsymbol{C}^{\mathrm{E}}(\underline{\boldsymbol{A}}, \boldsymbol{x})$ given by (4.4). The stresses $\left\{\sigma^{\nu_{j}}\right\}_{j=1}^{\infty}$ converge weakly in $L^{2}(\Omega)^{3 \times 3}$ to the homogenized stress $\sigma^{\mathrm{M}}$ given by $\sigma^{\mathrm{M}}=\boldsymbol{C}^{\mathrm{E}}(\underline{\boldsymbol{A}}, x) \epsilon\left(\boldsymbol{u}^{\mathrm{M}}\right)$ and the displacements $\left\{\boldsymbol{u}^{\nu_{j}}\right\}_{j=1}^{\infty}$ converge weakly to $\boldsymbol{u}^{\mathrm{M}}$ in $H_{0}^{1}(\Omega)^{3}$. Here $\boldsymbol{u}^{\mathrm{M}}$ is the solution of the homogenized problem given by

$$
-\operatorname{div}\left(\boldsymbol{C}^{\mathrm{E}}(\underline{\boldsymbol{A}}, \boldsymbol{x}) \epsilon\left(\boldsymbol{u}^{\mathrm{M}}\right)\right)=\boldsymbol{f}
$$

and $\boldsymbol{u}^{\mathrm{M}}=0$ on the boundary of $\Omega$. The effective compliance is given by $\left(\boldsymbol{C}^{\mathrm{E}}(\underline{\boldsymbol{A}}, x)\right)^{-1}$ and is denoted by $\boldsymbol{S}^{\mathrm{E}}(\underline{\boldsymbol{A}}, x)$.

We track the behaviour of the local stresses in each phase in the fine-scale limit. Consider the set $S_{t, i}^{\nu_{j}}$ of points in the $i$ th phase where $\Pi\left(\sigma^{\nu_{j}}\right)>t$. Passing to a subsequence if necessary, one has the asymptotic density of states $\theta_{t, i}(\boldsymbol{x})$ taking values in the unit interval. Following $\S 3$ we provide a link between $\theta_{t, i}$ and the macroscopic stress $\sigma^{\mathrm{M}}$ appearing in the homogenized composite. We introduce the tensor $\boldsymbol{F}^{i}(\boldsymbol{x}, \boldsymbol{y})$ defined for every constant symmetric $3 \times 3$ tensor $\bar{\epsilon}$ by

$$
\boldsymbol{F}^{i}(\boldsymbol{x}, \boldsymbol{y}) \bar{\epsilon}: \bar{\epsilon}=\chi_{i}(\boldsymbol{x}, \boldsymbol{y}) \Pi\left(\boldsymbol{A}^{i}\left(\epsilon_{\boldsymbol{y}}(\boldsymbol{w}(\boldsymbol{x}, \boldsymbol{y}))+\bar{\epsilon}\right)\right)
$$

where, for each $\boldsymbol{x}$, in $\Omega$ the function $w(\boldsymbol{x}, \boldsymbol{y})$ is the $Q$-periodic solution of (4.5). The microstress modulation function is given by

$$
f^{i}\left(\boldsymbol{x}, \boldsymbol{S}^{\mathrm{E}}(\underline{\boldsymbol{A}}, \boldsymbol{x}) \sigma^{\mathrm{M}}(\boldsymbol{x})\right)=\left\|\boldsymbol{F}^{i}(\boldsymbol{x}, \cdot) \boldsymbol{S}^{\mathrm{E}}(\underline{\boldsymbol{A}}, \boldsymbol{x}) \sigma^{\mathrm{M}}(\boldsymbol{x}): \boldsymbol{S}^{\mathrm{E}}(\underline{\boldsymbol{A}}, \boldsymbol{x}) \sigma^{\mathrm{M}}(\boldsymbol{x})\right\|_{L^{\infty}(Q)} .
$$

We then have the following theorem.

Theorem 4.2 (the homogenization constraint). If we consider a sequence $\left\{\boldsymbol{C}_{0}^{\kappa_{j}}\left(\underline{\boldsymbol{A}}, \boldsymbol{x}, \boldsymbol{x} \nu_{j}\right)\right\}_{j=1}^{\infty}$ with the associated $2 N+1$-tuple

$\left(\boldsymbol{C}^{\mathrm{E}}(\underline{\boldsymbol{A}}, \boldsymbol{x}) ; \theta_{t, 1}(\boldsymbol{x}), \ldots, \theta_{t, N}(\boldsymbol{x}) ; f^{1}\left(\boldsymbol{x}, \boldsymbol{S}^{\mathrm{E}}(\underline{\boldsymbol{A}}, \boldsymbol{x}) \sigma^{\mathrm{M}}(\boldsymbol{x})\right), \ldots, f^{N}\left(\boldsymbol{x}, \boldsymbol{S}^{\mathrm{E}}(\underline{\boldsymbol{A}}, \boldsymbol{x}) \sigma^{\mathrm{M}}(\boldsymbol{x})\right)\right)$,

then we have

$$
\theta_{t, i}(\boldsymbol{x})\left(f^{i}\left(\boldsymbol{x}, \boldsymbol{S}^{\mathrm{E}}(\underline{\boldsymbol{A}}, \boldsymbol{x}) \sigma^{\mathrm{M}}(\boldsymbol{x})\right)-t\right) \geqslant 0, \quad i=1, \ldots, N,
$$

almost everywhere on $\Omega$.

The next corollary is an immediate consequence of theorem 4.2. 
Corollary 4.3. Given the hypothesis of theorem 4.2, suppose that

$$
t>f^{i}\left(\boldsymbol{x}, \boldsymbol{S}^{\mathrm{E}}(\underline{\boldsymbol{A}}, \boldsymbol{x}) \sigma^{\mathrm{M}}(\boldsymbol{x})\right)
$$

on a set $\omega$, where $\omega \subset \Omega$. The stress in the $i$ th phase then satisfies $\Pi\left(\sigma^{\nu_{j}}\right) \leqslant t$ almost everywhere on $\omega$ outside $S_{t, i}^{\nu_{j}} \cap \omega$. Moreover the measure of the set $S_{t, i}^{\nu_{j}} \cap \omega$ tends to zero in the fine-scale limit.

We denote the set of points in $\Omega$ where $\Pi\left(\sigma^{\nu_{j}}\right)>t$ by $S_{t}^{\nu_{j}}$, and one has the following corollary.

Corollary 4.4. Given the hypothesis of theorem 4.2, suppose that

$$
t>f^{i}\left(\boldsymbol{x}, \boldsymbol{S}^{\mathrm{E}}(\underline{\boldsymbol{A}}, \boldsymbol{x}) \sigma^{\mathrm{M}}(\boldsymbol{x})\right)
$$

for $i=1, \ldots, N$ on a set $\omega$, where $\omega \subset \Omega$. Then $\Pi\left(\sigma^{\nu_{j}}\right) \leqslant t$ almost everywhere on $\omega$ outside $S_{t}^{\nu_{j}} \cap \omega$, moreover the measure of the set $S_{t}^{\nu_{j}} \cap \omega$ tends to zero in the fine-scale limit.

As in the general case, one finds that a pointwise bound on the macrostress modulation function delivers a pointwise bound on the equivalent stress in the composite when the microstructure is sufficiently fine relative to the size of the domain and variation of the body force.

Corollary 4.5 (pointwise bounds on the equivalent stress). Given the hypothesis of theorem 4.2, suppose that

$$
t>f^{i}\left(\boldsymbol{x}, \boldsymbol{S}^{\mathrm{E}}(\underline{\boldsymbol{A}}, \boldsymbol{x}) \sigma^{\mathrm{M}}(\boldsymbol{x})\right)
$$

for $i=1, \ldots, N$ on a set $\omega$, where $\omega \subset \Omega$. Then one can pass to a subsequence if necessary to find that there is a critical $\varepsilon_{0}$ such that for every $\varepsilon_{j}<\varepsilon_{0}$

$$
\Pi\left(\sigma^{\varepsilon_{j}}(\boldsymbol{x})\right) \leqslant t
$$

for almost every $\boldsymbol{x}$ in $\omega$. Here $\varepsilon_{0}$ can depend upon position.

Set

$$
\tilde{t}=\sup _{\boldsymbol{x} \in \omega}\left(\max _{i}\left\{f^{i}\left(\boldsymbol{x}, \boldsymbol{S}^{\mathrm{E}}(\underline{\boldsymbol{A}}, \boldsymbol{x}) \sigma^{\mathrm{M}}(\boldsymbol{x})\right)\right\}\right) .
$$

From corollary 4.5 one then has a critical scale $\varepsilon_{0}$ (depending possibly on $\boldsymbol{x}$ ) such that for every $\varepsilon_{j}<\varepsilon_{0}$

$$
\Pi\left(\sigma^{\varepsilon_{j}}(\boldsymbol{x})\right) \leqslant \tilde{t}
$$

for almost all points in $\omega$.

The following corollary provides an upper bound on the limit superior of the $L^{\infty}$ norms of local stresses in the fine-scale limit.

Corollary 4.6. Suppose it is known that $\ell=\limsup _{j \rightarrow \infty}\left\|\chi_{i}^{\nu_{j}} \Pi\left(\sigma^{\nu_{j}}\right)\right\|_{\infty}<\infty$. Suppose further that, for an interval $0<\delta<b$, the measure (volume) of the sets where $\theta_{\ell-\delta, i}>0$ does not vanish. Then

$$
\limsup _{j \rightarrow \infty}\left\|\chi_{i}^{\nu_{j}} \Pi\left(\sigma^{\nu_{j}}\right)\right\|_{\infty} \leqslant\left\|f^{i}\left(\boldsymbol{x}, \boldsymbol{S}^{\mathrm{E}}(\underline{\boldsymbol{A}}, \boldsymbol{x}) \sigma^{\mathrm{M}}(\boldsymbol{x})\right)\right\|_{\infty} .
$$

Corollary 4.6 follows upon setting $t=\ell-\delta$ and applying (3.14).

For $1 \leqslant p<\infty$ we provide bounds on $\lim \sup _{j \rightarrow \infty}\left\|\chi_{i}^{\nu_{j}} \Pi\left(\sigma^{\nu_{j}}\right)\right\|_{p}$. 
Corollary 4.7. Given that $\limsup _{j \rightarrow \infty}\left\|\chi_{i}^{\nu_{j}} \Pi\left(\sigma^{\epsilon_{j}}\right)\right\|_{\infty}<\infty$,

$$
\limsup _{j \rightarrow \infty}\left\|\chi_{i}^{\nu_{j}} \Pi\left(\sigma^{\nu_{j}}\right)\right\|_{p} \leqslant\left\|f^{i}\left(\boldsymbol{x}, \boldsymbol{S}^{\mathrm{E}}(\underline{\boldsymbol{A}}, \boldsymbol{x}) \sigma^{\mathrm{M}}(\boldsymbol{x})\right)\right\|_{\infty}|\Omega|^{1 / p}
$$

for any $p, 1 \leqslant p<\infty$.

This result follows from theorem 4.2 using the same arguments given to establish corollary 3.6.

\section{(a) Proofs of theorems 4.1 and 4.2}

To expedite the proofs we write the elements of the sequence $\left\{\boldsymbol{C}_{0}^{\kappa_{j}}\left(\underline{\boldsymbol{A}}, \boldsymbol{x}, \boldsymbol{x} \nu_{j}\right)\right\}_{j=1}^{\infty}$ in terms of characteristic functions. For the partition of $\Omega$ given by $\bigcup_{\ell=1} O_{\ell}^{\kappa_{j}}$ for which the diameters of $O_{\ell}^{\kappa_{j}}$ are less than $\kappa_{j}$, we introduce the characteristic functions $\chi_{\ell}^{\kappa_{j}}$ such that $\chi_{\ell}^{\kappa_{j}}(\boldsymbol{x})=1$ for $\boldsymbol{x}$ in $O_{\ell}^{\kappa_{j}}$ and zero otherwise. Then

$$
\boldsymbol{C}_{0}^{\kappa_{j}}\left(\underline{\boldsymbol{A}}, \boldsymbol{x}, \boldsymbol{x} \nu_{j}\right)=\sum_{i}^{N} \chi_{i}^{j}\left(\boldsymbol{x}, \nu_{j} \boldsymbol{x}\right) \boldsymbol{A}_{i},
$$

where the characteristic function of the $i$ th phase in the composite is specified by

$$
\chi_{i}^{j}(\boldsymbol{x}, \boldsymbol{y})=\sum_{\ell} \chi_{\ell}^{\kappa_{j}}(\boldsymbol{x}) \chi_{i}\left(\boldsymbol{x}_{\kappa_{j}}^{\ell}, \boldsymbol{y}\right)
$$

We start with the proof of theorem 4.2. Consider the set of points $S_{t, i}^{\nu_{j}}$ in the $i$ th phase for which $\Pi\left(\sigma^{\nu_{j}}\right)>t$ for a fixed positive number $t$. The characteristic function for $S_{t, i}^{\nu_{j}}$ is denoted by $\chi_{t, i}^{j}(\boldsymbol{x})$. Passing to a subsequence if necessary, one has that $\chi_{t, i}^{j} \stackrel{*}{\rightarrow} \theta_{t, i}$ in $L^{\infty}(\Omega)$ as $j \rightarrow \infty$. Here the density $\theta_{t, i}(\boldsymbol{x})$ takes values in $[0,1]$. For any non-negative smooth test function $p(\boldsymbol{x})$ one has

$$
t \int_{\Omega} p \chi_{t, i}^{j} \mathrm{~d} \boldsymbol{x} \leqslant \int_{\Omega} p \chi_{t, i}^{j} \Pi\left(\sigma^{\nu_{j}}\right) \mathrm{d} \boldsymbol{x} .
$$

Taking the limit on the left-hand side of (4.19) gives

$$
t \int_{\Omega} p \theta_{t, i} \mathrm{~d} \boldsymbol{x}
$$

As before the goal is to pass to the limit on the right-hand side of (4.19) to obtain a pointwise upper bound on the product $t \theta_{t, i}$. This will follow from the following lemma.

Lemma 4.8. There exists a subsequence of $\left\{\chi_{t, i}^{j}\right\}_{j=1}^{\infty}$ and $\left\{\sigma^{\nu_{j}}\right\}_{j=1}^{\infty}$, also denoted by $\left\{\chi_{t, i}^{j}\right\}_{j=1}^{\infty}$ and $\left\{\sigma^{\nu_{j}}\right\}_{j=1}^{\infty}$, such that

$$
\chi_{t, i}^{j} \Pi\left(\sigma^{\nu_{j}}\right) \rightarrow \boldsymbol{A}_{i} \boldsymbol{\Pi} \boldsymbol{A}_{i} \boldsymbol{H}_{t}(\boldsymbol{x}) \boldsymbol{S}^{\mathrm{E}}(\underline{\boldsymbol{A}}, \boldsymbol{x}) \sigma^{\mathrm{M}}(\boldsymbol{x}): \boldsymbol{S}^{\mathrm{E}}(\underline{\boldsymbol{A}}, \boldsymbol{x}) \sigma^{\mathrm{M}}(\boldsymbol{x})
$$

in the sense of distributions. Here $\boldsymbol{A}_{i} \boldsymbol{\Pi} \boldsymbol{A}_{i} \boldsymbol{H}_{t}(\boldsymbol{x})$ is given by

$$
\boldsymbol{A}_{i} \boldsymbol{\Pi} \boldsymbol{A}_{i} \boldsymbol{H}_{t}(\boldsymbol{x}) \bar{\epsilon}: \bar{\epsilon}=\lim _{r \rightarrow 0} \lim _{j \rightarrow \infty}\left(\frac{1}{|Q(\boldsymbol{x}, r)|}\right) \int_{Q(\boldsymbol{x}, r)} \chi_{t, i}^{j} \Pi\left(\boldsymbol{A}_{i}\left(\epsilon\left(\boldsymbol{w}_{\bar{\epsilon}}^{j, r}\right)+\bar{\epsilon}\right)\right) \mathrm{d} \boldsymbol{y},
$$

where $\bar{\epsilon}$ is any constant strain and the local oscillatory response function is denoted by $\boldsymbol{w}_{\bar{\epsilon}}^{j, r}$. Here $\boldsymbol{w}_{\bar{\epsilon}}^{j, r}$ is the $Q(\boldsymbol{x}, r)$-periodic solution of

$$
-\operatorname{div}\left(\boldsymbol{C}_{0}^{\kappa_{j}}\left(\underline{\boldsymbol{A}}, \boldsymbol{y}, \boldsymbol{y} \nu_{j}\right)\left(\epsilon\left(\boldsymbol{w}_{\bar{\epsilon}}^{j, r}(\boldsymbol{y})\right)+\bar{\epsilon}\right)\right)=0
$$

for $\boldsymbol{y}$ in $Q(\boldsymbol{x}, r)$. 
This lemma is established in the same way as lemma 3.7.

Passing to subsequences according to lemma 4.8 and on taking limits in (4.19) it follows that

$$
\boldsymbol{A}_{i} \boldsymbol{\Pi} \boldsymbol{A}_{i} \boldsymbol{H}_{t}(\boldsymbol{x}) \boldsymbol{S}^{\mathrm{E}}(\underline{\boldsymbol{A}}, \boldsymbol{x}) \sigma^{\mathrm{M}}(\boldsymbol{x}): \boldsymbol{S}^{\mathrm{E}}(\underline{\boldsymbol{A}}, \boldsymbol{x}) \sigma^{\mathrm{M}}(\boldsymbol{x})-t \theta_{t, i}(\boldsymbol{x}) \geqslant 0,
$$

almost everywhere on $\Omega$. Passing to a diagonal sequence we have $\lim _{j \rightarrow \infty} r_{j}=0$, $\lim _{j \rightarrow \infty} \kappa_{j} / r_{j}=0, \lim _{j \rightarrow \infty}\left(1 / \nu_{j}\right) \kappa_{j}=0$,

$$
\boldsymbol{A}_{i} \boldsymbol{\Pi} \boldsymbol{A}_{i} \boldsymbol{H}_{t}(\boldsymbol{x}) \bar{\epsilon}: \bar{\epsilon}=\lim _{j \rightarrow \infty}\left(\frac{1}{\left|Q\left(\boldsymbol{x}, r_{j}\right)\right|}\right) \int_{Q\left(\boldsymbol{x}, r_{j}\right)} \chi_{t, i}^{j} \Pi\left(\boldsymbol{A}_{i}\left(\epsilon\left(\boldsymbol{w}_{\bar{\epsilon}}^{j, r_{j}}\right)+\bar{\epsilon}\right)\right) \mathrm{d} \boldsymbol{y}
$$

and

$$
\lim _{j \rightarrow \infty}\left(\frac{1}{\left|Q\left(\boldsymbol{x}, r_{j}\right)\right|}\right) \int_{Q\left(\boldsymbol{x}, r_{j}\right)} \chi_{t, i}^{j} \mathrm{~d} \boldsymbol{x}=\theta_{t, i}(\boldsymbol{x}) .
$$

Here $\boldsymbol{w}_{\bar{\epsilon}}^{j, r_{j}}$ solves (4.21) with $r=r_{j}$. One can arrange things so that the cubes $Q\left(\boldsymbol{x}, r_{j}\right)$ contain the point $\boldsymbol{x}$ and an integral number of periods of side length $1 / \nu_{j}$. We change coordinates by translation and re-scaling so that $Q\left(\boldsymbol{x}, r_{j}\right)$ is transformed to the unit cube $Q$ centred at the origin. The change of variables is given by $\boldsymbol{y}=\boldsymbol{x}_{0}^{j}+\boldsymbol{z r}_{j}$, where $\boldsymbol{x}_{0}^{j}$ is the centre point of $Q\left(\boldsymbol{x}, r_{j}\right)$ and $\boldsymbol{z}$ lies in $Q$. One can write $\boldsymbol{w}_{\bar{\epsilon}}^{j, r_{j}}(\boldsymbol{y})=r_{j} \phi^{j}\left(\left(\boldsymbol{y}-\boldsymbol{x}_{0}^{j}\right) / r_{j}\right)$, where $\phi^{j}(\boldsymbol{z})$ is the $Q$-periodic solution of

$$
-\operatorname{div}\left(\boldsymbol{C}_{0}^{\kappa_{j}}\left(\underline{\boldsymbol{A}}, \boldsymbol{x}_{0}^{j}+r_{j} \boldsymbol{z}, r_{j} \nu_{j} \boldsymbol{z}\right)\left(\epsilon\left(\phi^{j}(\boldsymbol{z})\right)+\bar{\epsilon}\right)\right)=0, \quad \text { for } \boldsymbol{z} \text { in } Q .
$$

Under this change of coordinates $\chi_{t, i}^{j}=\chi_{t, i}^{j}\left(\boldsymbol{x}_{0}^{j}+r_{j} \boldsymbol{z}\right)$ and

$$
\boldsymbol{A}_{i} \boldsymbol{\Pi} \boldsymbol{A}_{i} \boldsymbol{H}_{t}(\boldsymbol{x}) \bar{\epsilon}: \bar{\epsilon}=\lim _{j \rightarrow \infty} \int_{Q} \chi_{t, i}^{j}\left(\boldsymbol{x}_{0}^{j}+r_{j} \boldsymbol{z}\right) \Pi\left(\boldsymbol{A}_{i}\left(\epsilon\left(\phi^{j}(\boldsymbol{z})\right)+\bar{\epsilon}\right)\right) \mathrm{d} \boldsymbol{z} .
$$

Now introduce $\boldsymbol{C}_{0}\left(\underline{\boldsymbol{A}}, \boldsymbol{x}, r_{j} \nu_{j} \boldsymbol{z}\right)=\sum_{i} \boldsymbol{A}_{i} \chi_{i}\left(\boldsymbol{x}, r_{j} \nu_{j} \boldsymbol{z}\right)$ and the $Q$-periodic solutions $\tilde{\phi}^{j}$ of

$$
-\operatorname{div}\left(\boldsymbol{C}_{0}\left(\underline{\boldsymbol{A}}, \boldsymbol{x}, r_{j} \nu_{j} \boldsymbol{z}\right)\left(\epsilon\left(\tilde{\phi}^{j}(\boldsymbol{z})\right)+\bar{\epsilon}\right)\right)=0, \text { for } \boldsymbol{z} \text { in } Q .
$$

Recalling (4.2) it follows that

$$
\lim _{j \rightarrow \infty} \int_{Q}\left|\boldsymbol{C}_{0}^{\kappa_{j}}\left(\underline{\boldsymbol{A}}, \boldsymbol{x}_{0}^{j}+r_{j} \boldsymbol{z}, r_{j} \nu_{j} \boldsymbol{z}\right)-\boldsymbol{C}_{0}\left(\underline{\boldsymbol{A}}, \boldsymbol{x}, r_{j} \nu_{j} \boldsymbol{z}\right)\right| \mathrm{d} \boldsymbol{z}=0 .
$$

Thus, application of the higher integrability theorem of Meyers \& Elcrat (1975) (see Bensoussan et al. 1978) shows that

$$
\lim _{j \rightarrow \infty} \int_{Q}\left|\epsilon\left(\phi^{j}(\boldsymbol{z})\right)-\epsilon\left(\tilde{\phi}^{j}(\boldsymbol{z})\right)\right|^{2} \mathrm{~d} \boldsymbol{z}=0 .
$$

Hence

$$
\boldsymbol{A}_{i} \boldsymbol{\Pi} \boldsymbol{A}_{i} \boldsymbol{H}_{t}(\boldsymbol{x}) \bar{\epsilon}: \bar{\epsilon}=\lim _{j \rightarrow \infty} \int_{Q} \chi_{t, i}^{j}\left(\boldsymbol{x}_{0}^{j}+r_{j} \boldsymbol{z}\right) \Pi\left(\boldsymbol{A}_{i}\left(\epsilon\left(\tilde{\phi}^{j}(\boldsymbol{z})\right)+\bar{\epsilon}\right)\right) \mathrm{d} \boldsymbol{z} .
$$

One can write $\tilde{\phi}^{j}(\boldsymbol{z})=\left(1 /\left(r_{j} \nu_{j}\right)\right) \varphi\left(r_{j} \nu_{j} \boldsymbol{z}\right)$, where $\varphi(\boldsymbol{s})$ is the $Q$-periodic solution of

$$
-\operatorname{div}\left(\boldsymbol{C}_{0}(\underline{\boldsymbol{A}}, \boldsymbol{x}, \boldsymbol{s})(\epsilon(\varphi(\boldsymbol{s}))+\bar{\epsilon})\right)=0, \quad \text { for } \boldsymbol{s} \text { in } Q .
$$


It is noted that the sequence $\left\{\epsilon\left(\tilde{\phi}^{j}(\boldsymbol{z})\right)\right\}_{j=1}^{\infty}$ is equi-integrable, since $\epsilon\left(\tilde{\phi}^{j}(\boldsymbol{z})\right)=$ $\epsilon\left(\varphi\left(r_{j} \nu_{j} \boldsymbol{z}\right)\right)$. Writing

$$
\chi_{i}^{j}\left(\boldsymbol{x}_{0}^{j}+r_{j} \boldsymbol{z}, r_{j} \nu_{j} \boldsymbol{z}\right)=\chi_{i}\left(\boldsymbol{x}, r_{j} \nu_{j} \boldsymbol{z}\right)+\chi_{i}^{j}\left(\boldsymbol{x}_{0}^{j}+r_{j} \boldsymbol{z}, r_{j} \nu_{j} \boldsymbol{z}\right)-\chi_{i}\left(\boldsymbol{x}, r_{j} \nu_{j} \boldsymbol{z}\right)
$$

one notes from the equi-integrability of $\left\{\epsilon\left(\tilde{\phi}^{j}(\boldsymbol{z})\right)\right\}_{j=1}^{\infty}$ and (4.28) that

$$
\boldsymbol{A}_{i} \boldsymbol{\Pi} \boldsymbol{A}_{i} \boldsymbol{H}_{t}(\boldsymbol{x}) \bar{\epsilon}: \bar{\epsilon}=\lim _{j \rightarrow \infty} \int_{Q} \chi_{t, i}^{j}\left(\boldsymbol{x}_{0}^{j}+r_{j} \boldsymbol{z}\right) \chi_{i}\left(\boldsymbol{x}, r_{j} \nu_{j} \boldsymbol{z}\right) \Pi\left(\boldsymbol{A}_{i}\left(\epsilon\left(\tilde{\phi}^{j}(\boldsymbol{z})\right)+\bar{\epsilon}\right)\right) \mathrm{d} \boldsymbol{z} .
$$

Application of Hölder's inequality gives

$$
\begin{aligned}
\boldsymbol{A}_{i} \boldsymbol{\Pi} & \boldsymbol{A}_{i} \boldsymbol{H}_{t}(\boldsymbol{x}) \bar{\epsilon}: \bar{\epsilon} \\
& \leqslant\left(\limsup _{j \rightarrow \infty}\left(\frac{1}{|Q|}\right) \int_{Q} \chi_{t, i}^{j}\left(\boldsymbol{x}_{0}^{j}+r_{j} \boldsymbol{z}\right) \mathrm{d} \boldsymbol{z}\right)\left\|\chi_{i}(\boldsymbol{x}, \cdot) \Pi\left(\boldsymbol{A}_{i}(\epsilon(\varphi(\cdot))+\bar{\epsilon})\right)\right\|_{L^{\infty}(Q)} \\
& =\theta_{t, i}(\boldsymbol{x})\left\|\chi_{i}(\boldsymbol{x}, \cdot) \Pi\left(\boldsymbol{A}_{i}(\epsilon(\varphi(\cdot))+\bar{\epsilon})\right)\right\|_{L^{\infty}(Q)} .
\end{aligned}
$$

Here we recall that both $\tilde{\phi}^{j}$ and $\varphi$ are parametrized by $\boldsymbol{x}$ and theorem 4.2 follows from (4.22). The proof of theorem 4.2 for the case of piecewise constant locally periodic microstructures is simpler, since $\phi^{j}=\tilde{\phi}^{j}$ for sufficiently small cubes $Q\left(\boldsymbol{x}, r_{j}\right)$.

We give the proof of theorem 4.1. We consider a sequence of continuously graded locally periodic microstructures with elasticity tensors $\left\{\boldsymbol{C}_{0}^{\kappa_{j}}\left(\underline{\boldsymbol{A}}, \boldsymbol{x}, \boldsymbol{x} \nu_{j}\right)\right\}_{j=1}^{\infty}$ given by (4.17). We prove that this sequence G-converges to $\boldsymbol{C}^{\mathrm{E}}(\underline{\boldsymbol{A}}, \boldsymbol{x})$ given by (4.4). From the theory of G-convergence we can pass to a subsequence also denoted by $\left\{\boldsymbol{C}_{0}^{\kappa_{j}}\left(\underline{\boldsymbol{A}}, \boldsymbol{x}, \boldsymbol{x} \nu_{j}\right)\right\}_{j=1}^{\infty}$ that G-converges to $\widetilde{\boldsymbol{C}}^{\mathrm{E}}(\underline{\boldsymbol{A}}, \boldsymbol{x})$. Our first goal is to show that

$$
\widetilde{\boldsymbol{C}}^{\mathrm{E}}(\underline{\boldsymbol{A}}, \boldsymbol{x})=\boldsymbol{C}^{\mathrm{E}}(\underline{\boldsymbol{A}}, \boldsymbol{x}) .
$$

To do this we use (2.10) to write

$$
\begin{aligned}
& \widetilde{\boldsymbol{C}}^{\mathrm{E}}(\underline{\boldsymbol{A}}, \boldsymbol{x}) \bar{\epsilon}: \bar{\epsilon} \\
& \quad=\lim _{r \rightarrow 0} \lim _{j \rightarrow \infty}\left(\frac{1}{|Q(\boldsymbol{x}, r)|}\right) \int_{Q(\boldsymbol{x}, r)} \boldsymbol{C}_{0}^{\kappa_{j}}\left(\underline{\boldsymbol{A}}, \boldsymbol{y}, \boldsymbol{y} \nu_{j}\right)\left(\epsilon\left(\boldsymbol{w}_{\bar{\epsilon}}^{j, r}\right)+\bar{\epsilon}\right):\left(\epsilon\left(\boldsymbol{w}_{\bar{\epsilon}}^{j, r}\right)+\bar{\epsilon}\right) \mathrm{d} \boldsymbol{y},
\end{aligned}
$$

where $\boldsymbol{w}_{\bar{\epsilon}}^{j, r}$ solves (4.21). Passing to a diagonal sequence, we have

$$
\lim _{j \rightarrow \infty} r_{j}=0, \quad \lim _{j \rightarrow \infty} \frac{\kappa_{j}}{r_{j}}=0, \quad \lim _{j \rightarrow \infty}\left(\frac{1}{\nu_{j}}\right) / \kappa_{j}=0
$$

and

$$
\begin{aligned}
& \widetilde{\boldsymbol{C}}^{\mathrm{E}}(\underline{\boldsymbol{A}}, \boldsymbol{x}) \bar{\epsilon}: \bar{\epsilon} \\
& \quad=\lim _{j \rightarrow \infty}\left(\frac{1}{\left|Q\left(\boldsymbol{x}, r_{j}\right)\right|}\right) \int_{Q\left(\boldsymbol{x}, r_{j}\right)} \boldsymbol{C}_{0}^{\kappa_{j}}\left(\underline{\boldsymbol{A}}, \boldsymbol{y}, \boldsymbol{y} \nu_{j}\right)\left(\epsilon\left(\boldsymbol{w}_{\bar{\epsilon}}^{j, r_{j}}\right)+\bar{\epsilon}\right):\left(\epsilon\left(\boldsymbol{w}_{\bar{\epsilon}}^{j, r_{j}}\right)+\bar{\epsilon}\right) \mathrm{d} \boldsymbol{y} .
\end{aligned}
$$

Here $\boldsymbol{w}_{\bar{\epsilon}}^{j, r_{j}}$ solves (4.21) with $r=r_{j}$. As before, one can arrange things so that the cubes $Q\left(\boldsymbol{x}, r_{j}\right)$ contain the point $\boldsymbol{x}$ and an integral number of periods of side length $1 / \nu_{j}$. We change coordinates by translation and rescaling so that $Q\left(\boldsymbol{x}, r_{j}\right)$ is transformed to the unit cube $Q$ centred at the origin. The change of variables is given 
by $\boldsymbol{y}=\boldsymbol{x}_{0}^{j}+\boldsymbol{z} r_{j}$, where $\boldsymbol{x}_{0}^{j}$ is the centre point of $Q\left(\boldsymbol{x}, r_{j}\right)$ and $\boldsymbol{z}$ lies in $Q$. One writes $\boldsymbol{w}_{\bar{\epsilon}}^{j, r_{j}}(\boldsymbol{y})=r_{j} \phi^{j}\left(\left(\boldsymbol{y}-\boldsymbol{x}_{0}^{j}\right) / r_{j}\right)$, where $\phi^{j}(\boldsymbol{z})$ is the solution of (4.25). This gives

$$
\widetilde{\boldsymbol{C}}^{\mathrm{E}}(\underline{\boldsymbol{A}}, \boldsymbol{x}) \bar{\epsilon}: \bar{\epsilon}=\lim _{j \rightarrow \infty} \int_{Q} \boldsymbol{C}_{0}^{\kappa_{j}}\left(\underline{\boldsymbol{A}}, \boldsymbol{x}_{0}^{j}+\boldsymbol{z} r_{j}, r_{j} \nu_{j} \boldsymbol{z}\right)\left(\epsilon\left(\phi^{j}\right)+\bar{\epsilon}\right):\left(\epsilon\left(\phi^{j}\right)+\bar{\epsilon}\right) \mathrm{d} \boldsymbol{z} .
$$

We introduce $\boldsymbol{C}_{0}\left(\underline{\boldsymbol{A}}, \boldsymbol{x}, r_{j} \nu_{j} \boldsymbol{z}\right)=\sum_{i} \boldsymbol{A}_{i} \chi_{i}\left(\boldsymbol{x}, r_{j} \nu_{j} \boldsymbol{z}\right)$ and the solutions $\tilde{\phi}^{j}$ of $(4.27)$. Recalling (4.28), we see as before that

$$
\lim _{j \rightarrow \infty} \int_{Q}\left|\epsilon\left(\phi^{j}(\boldsymbol{z})\right)-\epsilon\left(\tilde{\phi}^{j}(\boldsymbol{z})\right)\right|^{2} \mathrm{~d} \boldsymbol{z}=0 .
$$

Thus from the equi-integrability of the sequence $\left\{\epsilon\left(\tilde{\phi}^{j}(\boldsymbol{z})\right)\right\}_{j=1}^{\infty}$ and a change of variables we deduce that $\widetilde{\boldsymbol{C}}^{\mathrm{E}}(\underline{\boldsymbol{A}}, \boldsymbol{x})=\boldsymbol{C}^{\mathrm{E}}(\underline{\boldsymbol{A}}, \boldsymbol{x})$. It is evident from the derivation given above that every subsequence of $\left\{\boldsymbol{C}_{0}^{\kappa}\left(\underline{\boldsymbol{A}}, \boldsymbol{x}, \boldsymbol{x} \nu_{j}\right)\right\}_{j=1}^{\infty}$ has a subsequence that Gconverges to the same G-limit given by (4.4). Since the topology of G-convergence is metrizable, we deduce that the whole sequence $\left\{\boldsymbol{C}_{0}^{\kappa_{j}}\left(\underline{\boldsymbol{A}}, \boldsymbol{x}, \boldsymbol{x} \nu_{j}\right)\right\}_{j=1}^{\infty} \mathrm{G}$-converges to (4.4). A standard application of the theorem of Meyers \& Elcrat (1975) shows that $\boldsymbol{C}^{\mathrm{E}}(\underline{\boldsymbol{A}}, \boldsymbol{x})$ is a continuous function of $\boldsymbol{x}$. Conversely, given an effective elastic tensor of the form (4.4), we can apply the procedure just used to construct a sequence of continuously graded locally periodic microstructures that G-converge to it.

This research effort is sponsored by NSF through grant DMS-0072469 and by the Air Force Office of Scientific Research, Air Force Materiel Command USAF, under grant number F49620-02-10041. The US Government is authorized to reproduce and distribute reprints for governmental purposes notwithstanding any copyright notation thereon. The views and conclusions herein are those of the authors and should not be interpreted as necessarily representing the official policies or endorsements, either expressed or implied, of the Air Force Office of Scientific Research or the US Government.

\section{References}

Bensoussan, A., Lions, J. L. \& Papanicolaou, G. 1978 Asymptotic analysis for periodic structures. Studies in Mathematics and its Applications, vol. 5. Amsterdam: North-Holland.

Biot, M. A. 1966 Fundamental skin effect in anisotropic solid mechanics. Int. J. Solids Struct. 2, 645-663.

Buryachenko, V. A. 2001 Multiparticle effective field and related methods in micromechanics of composite materials. Appl. Mech. Rev. 54, 1-47.

Dal Maso, G. 1993 An introduction to $\Gamma$-convegence. Progress in Nonlinear Differential Equations and Their Applications, vol. 8. Boston: Birkhäuser.

Duvaut, G. \& Lions, J. L. 1976 Inequalities in mechanics and physics. Grundlehren der mathematischen Wissenschaften in Einzeldarstellungen, vol. 219. Springer.

Evans, L. C. 1990 Weak convergence methods for nonlinear partial differential equations. CBMS Regional Conference Series in Mathematics, vol. 74. Providence, RI: American Mathematical Society.

Glasser, A. M. 1997 The use of transient FGM interlayers for joining advanced ceramics. Composites $B \mathbf{2 8}, 71-84$.

Koizumi, M. 1997 FGM activities in Japan. Composites B 28, 1-4.

Lipton, R. 2002 Relaxation through homogenization for optimal design problems with gradient constraints. J. Optim. Theory Applicat. 114, 27-53.

Lipton, R. 2003 Stress constrained G closure and relaxation of structural design problems. $Q$. Appl. Math. (In the press.) 
Meyers, N. G. \& Elcrat, A. 1975 Some results on regularity of non-linear elliptic equations and quasi-regular functions. Duke Math. J. 47, 121-136.

Milton, G. W. 2002 The theory of composites. Applied and Computational Mathematics, vol. 6. Cambridge University Press.

Murat, F. \& Tartar, L. 1997 H convergence. In Topics in the mathematical modelling of composite materials (ed. A. V. Cherkaev \& R. V. Kohn), pp. 21-43. Boston: Birkhäuser.

Pagano, N. \& Rybicky, E. F. 1974 On the significance of the effective modulus solutions for fibrous composites. J. Composite Mater. 8, 214-228.

Pagano, N. \& Yuan, F. G. 2000 The significance of the effective modulus theory (homogenization) in composite laminate mechanics. Composites Sci. Technol. 60, 2471-2488.

Ponte Castañeda, P. \& Suquet, P. 1998 Nonlinear composites. Adv. Appl. Mech. 34, 171-302.

Simon, L. 1979 On G-convergence of elliptic operators. Indiana Univ. Math. J. 28, 587-594.

Spagnolo, S. 1976 Convergence in energy for elliptic operators. In Proc. 3rd Symp. on Numerical Solutions of Partial Differential Equations (ed. B. Hubbard), pp. 469-498. Academic.

Tartar, L. 2000 An introduction to the homogenization method in optimal design. Springer Lecture Notes in Mathematics, vol. 1740, pp. 45-156.

Tsai, S. W. \& Hahn, H. T. 1980 Introduction to composite materials. Lancaster, PA: Technomic Publishing Company. 